\title{
Nonlinear vibration-damping investigation of a vertical cantilever beam simulation aircraft tail via NTDSC procedure
}

\author{
A.T.EL-Sayed ${ }^{\text {a,* }}$, H.S. Bauomy b,c \\ ${ }^{a}$ Department of Basic Sciences, Modern Academy for Engineering and Technology, Egypt \\ ${ }^{b}$ Department of Mathematics, College of Arts and Science in Wadi Addawasir, Prince Sattam \\ Bin Abdulaziz University, P.O. Box 54,Wadi Addawasir 11991, Saudi Arabia \\ ${ }^{c}$ Department of Mathematics, Faculty of Science, Zagazig University, Zagazig 44519, Egypt
}

\begin{abstract}
A nonlinear time delay saturation controller (NTDSC) used to minimize the vibrations of a vertical cantilever beam simulated as an airplane tail and exposed to harmonic force. A modified form of saturation-based controllers is given to lessen system vibrations. The perturbation technique is used to generate an approximate solution to evaluate the nonlinear behavior of this model. Time histories of the primary system and the controller are shown to demonstrate the reaction with and without control. The time-history response, as well as the impacts of the parameters on the system and controller, were simulated numerically using the MATLAB program. The RouthHurwitz criterion was used to show the stability investigation at simultaneous resonance ( $\Omega \cong \omega_{s}, \omega_{s} \cong 2 \omega_{c}$ ). The impact of controller parameters and time delays on system response curves are examined. Time-delays $\left(\tau_{1}, \tau_{2}\right.$ and $\left.\tau_{3}\right)$ are explored in the control loop for their implications on controller performance and system stability. The impacts of time delay are also investigated in order to establish the operation's safe zone. Validation curves are provided to show how closely the perturbation and numerical solutions are related. A comparison is made with recently released papers.
\end{abstract}

Keywords: Perturbation, Nonlinear time delay, Saturation controller, Stability, Sensor, Actuator, External excitation.

\section{Introduction}

In recent years, most control process built on the saturation phenomenon have been developed to reduce system vibrations. Many researchers studied this phenomenon in various dynamical systems. Oueini et al. [1] recommended an active control for a cantilever beam by adding a vibration damper. The flexible structure with the controller have been presented analytically using multiple scale method at two-to-one autoparametric resonances. Pai et al. [2] enhanced the theoretical, numerical, and experimental performance of the linear position feedback method and the nonlinear saturation controller (NSC). They discovered that NSC is effective at reducing steadystate vibrations in this study. Oueini and Nayfeh [3] a control strategy for the excited beam by cubic velocity feedback under a principal parametric resonance is suggested. The analysis detected that this controller reduced the amplitude of the response. Pai and Schulz [4] proposed a controller that was coupled to a system with quadratic terms that were dependent on the occurrence of saturation. They used PZT (Piezoelectric) patches as actuators and sensors to control the system's oscillation. Pai et al. [5] hypothesized that some absorbers rely on saturation processes to reduce vibrations in a

\footnotetext{
* A.T. EL-Sayed

e-mail: ashraftaha211@yahoo.com
} 
cantilevered skew aluminum plate model. For reducing the model's vibrations, Ashour and Nayfeh [6] developed a saturation-based absorber. They determine the frequency of excitation together with the system in order to increase the saturation control's action. Jun et al. [7] used conventional PZT patches to demonstrate the saturation controller to the structure. In contrast to a linear model, where raising the feedback gain value could lead to instability, these authors employed analytical methods to show that the nonlinear absorber (NSC) was globally stable. The power need for a nonlinear absorber, on the other hand, will be higher than for a linear system. Jun et al. [8] presented NSC for reducing self-excited plant vibrations. The control method employs quadratic nonlinearity to combine the absorber among the plants. The effect outcomes of the time-delay of a beam with NSC was proposed by Xu et al. [9]. In the analytical results, the multiple scales method revealed significantly more complex dynamics for the system. The frequency bandwidth of effective vibration suppuration was broadened or narrowed by the existence of the time-delay. The goal of Macro Fiber Composite (MFC) actuators, according to Mitura et al. [10], is to reduce beam or plate vibrations. The efficiency of NSC's vibration suppuration is investigated for a few model parameters. The system is extremely sensitive to changes in the parameters being studied. Warminski et al. [11] concentrated on appliance of special controllers for a flexible beam with MFC actuators. Mathematical solutions for the beam with NSC obtained using perturbation technique. The best controllers for reduction the vibration for the system are NSC and (Positive Position Feedback) PPF controllers. Saeed et al. [12] illustrated time-delay saturation controller to decrease oscillations of a nonlinear beam. Hamed, and Elagan [13] considered effeteness of NSC control algorithms for huge oscillation of the beam structure. NSC was utilized by Hamed and Amer [14] to lessen the oscillation amplitude of a structure made by composite beams. To lessen the horizontal vibration induced by a nonlinear differential equation simulation, Kamel et al. [15] proposed employing a magnetically levitated body with an NSC. For nonlinear oscillation reduction, Omidi and Mahmoodi [16] used Positive Position Feedback (PPF), Integral Resonant Control (IRC), and Nonlinear Integral Positive Position Feedback (NIPPF). To solve the system analytically, they used the multiple scales technique. The results show that suppression performance is mostly determined by the controller build.The effect of positive position feedback on a self-excited beam subjected to an external periodic force, as well as the effect of time-delay in the positive position feedback loop on a self-excited beam subjected to periodic forced stimulation at the resonant frequency, were explored by [17,18]. For rotating-beam vibration suppression, Warminski and Latalski [19] proposed a nonlinear saturation control technique. Warminski et al. [20] looked at how nonlinear saturation-based control affected a self-excited beam produced towards the primary resonance. Xu et al. [21] modified a nonlinear saturation controller by substituting a quadratic velocity coupling term with a time delay for the initial quadratic position coupling term, applying negative time-delay velocity feedback to the primary device, and using this controller to suppress high amplitude vibrations of a versatile, geometrically nonlinear beam-like structure. Many closed-loop control systems employ loop delays, also known as "time delays". Time delays are inevitable in any active control system as a result of calculating system states, processing control algorithms, control interfaces, transport delay, and actuation delay.

The control system's capabilities are limited by the existence of time delays. Due to measurement delays, the controller receives "outdated" information on process activity. 
Furthermore, the control operation cannot be applied "on time" due to actuation delays. As a consequence, the efficacy of compensating for disruption effects is harmed by temporal delay. Time delay cannot be ignored when designing control systems; it can reduce system performance and potentially cause instability. Controller design and operation become more complex as a result. Time delays can jeopardize the system's stability. As a result, researchers became interested in time-delayed control systems. Control issues in loop-delayed systems have received a lot of attention [22]. The effects of time delay were investigated in order to determine the system secure operating zone [23]. The horizontal vibration of a magnetically levitated body subjected to multi-force excitations was reduced using a time-delayed position feedback controller. The effect of time delays on the saturation regulation of first-mode vibration in a stainless-steel beam has been investigated [24]. Delayed feedback control has been used to inhibit or stabilize the vibration of the primary system in a two-degree-of-freedom dynamical system with a parametrically stimulated pendulum [25]. The authors of [26-32] used different controllers to investigate the vibration regulation of a number of devices with time delays. Mondal and Chatterjee [33] suggested the use of a velocity feedback dependent nonlinear resonant controller to monitor the free and forced self-excited vibration of a nonlinear beam. The main goal of this paper is to investigate how the NTDSC technique can be used to suppress largeamplitude vibrations in a vertical cantilever beam modeled after an airplane tail. The analytical result obtained by using the perturbation technique in the case of near simultaneous internal and primary resonance. The data obtained from the MATLAB Simulink model's direct numerical simulation is compared to data obtained from empirical results obtained using the multiple time-scale approach. The system's response is then checked for stability, and the control technique's output is evaluated. A numerical solution is used to represent and present the impact of all parameters on the vibrating device and the NTDSC system. The rest of the paper is organized as follows. The mathematical model of a vertical cantilever beam simulated as an airplane tail is introduced and solved in Section 2. In Section 3, the stability analysis is carried out analytically using the perturbation method. The analytical response for the forced vibration of the beam with and without control is determined in Section 4 using the multiple time scale perturbation procedure, which is assisted by a numerical simulation in Matlab Simulink. The impact of time delay in the feedback circuit is also investigated, and the controller parameters are re-optimized. The comparison of analytical and numerical solutions is prepared in Section 5. In addition, a comparison with recent studies in the area is made. Conclusions on the suggested controller's merits and limitations are discussed in Section 6.

\section{Governing equations and perturbation solution}

Equation motion of the main system obtained from Ref. [16] modified by using NTDSC system as follows:

$$
\begin{aligned}
& \ddot{x}(t)+\varepsilon \eta_{s} \dot{x}(t)+\omega_{s}^{2} x(t)+\varepsilon \alpha x^{3}(t)+\varepsilon \beta x(t) \dot{x}^{2}(t)+\varepsilon \gamma x^{2}(t) \dot{x}(t)=\varepsilon f \cos (\Omega t) \\
& +\varepsilon \gamma_{1} v^{2}\left(t-\tau_{1}\right) \\
& \ddot{v}(t)+\varepsilon \eta_{c} \dot{v}(t)+\omega_{c}^{2} v(t)=\varepsilon \gamma_{2} x\left(t-\tau_{2}\right) v\left(t-\tau_{3}\right)
\end{aligned}
$$

Where $\eta_{s}$ and $\eta_{c}$ are the main system's and NSC's linear damping coefficients, $f$ and $\Omega$ are the system's forcing amplitude and the frequency, $\omega_{s}$ and $\omega_{c}$ are the main system's and NTDSC's natural frequencies, $\alpha, \beta$ and $\gamma$ are nonlinear variables, $\gamma_{1}$ is 
control signal gain, $\gamma_{2}$ is feedback signal gain, $\tau_{1}, \tau_{2}, \tau_{3}$ are the time delays and $\varepsilon$ is a small perturbation value. Fig. 1 shows connection of NTDSC system to the main system in a closed figure which describing equations (1) and (2).

(a)
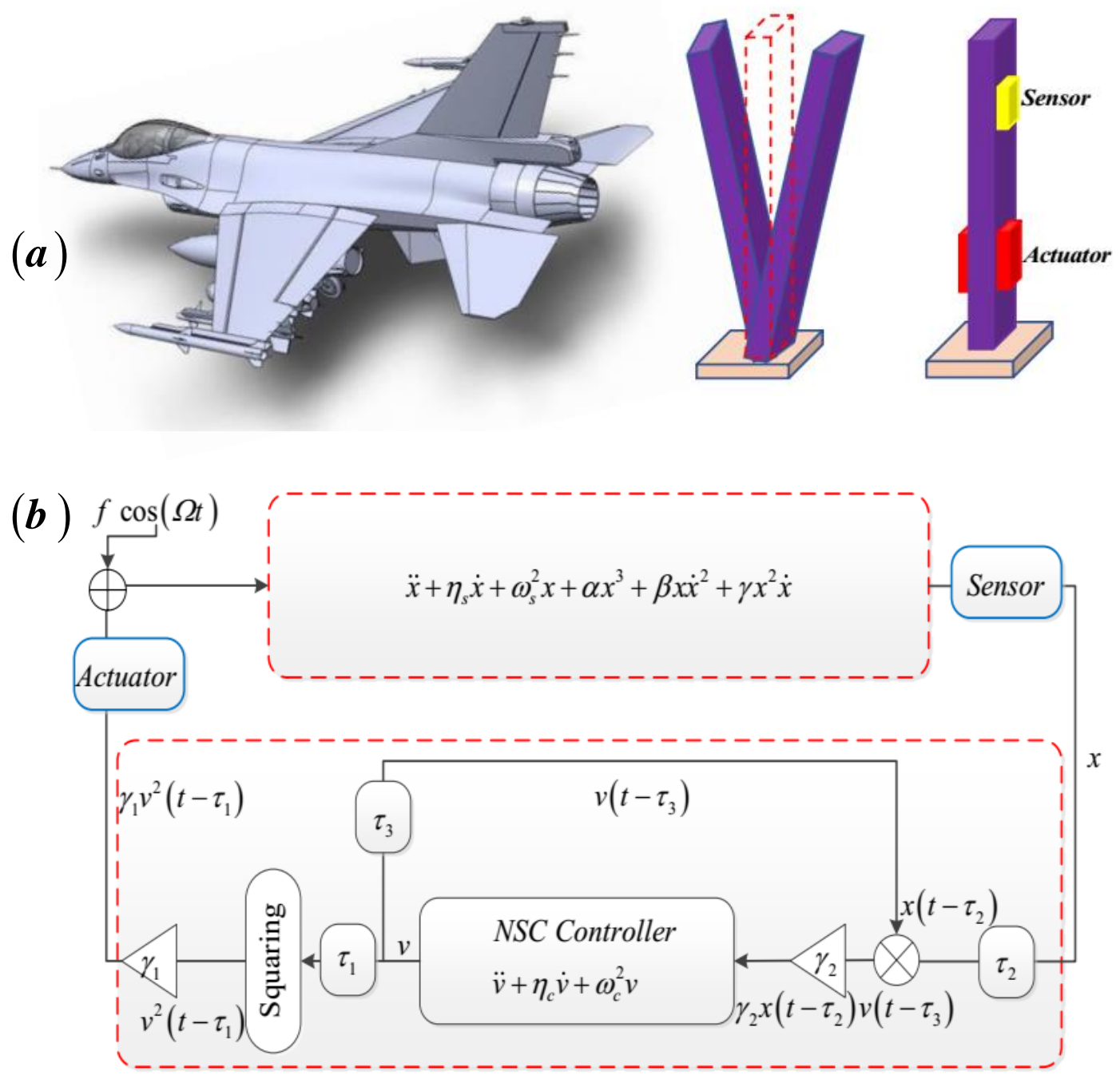

Fig. 1 (a) a vertical cantilever beam simulating aircraft tail, and (b) The block diagram of controlled system

\subsection{Mathematical Analysis}

In this section, the perturbation method $[34,35]$ is used to approximate the solution of equations (1) and (2) as follows:

$$
\begin{aligned}
& x(t, \varepsilon)=x_{0}\left(T_{0}, T_{1}\right)+\varepsilon x_{1}\left(T_{0}, T_{1}\right)+O\left(\varepsilon^{2}\right) \\
& v(t, \varepsilon)=v_{0}\left(T_{0}, T_{1}\right)+\varepsilon v_{1}\left(T_{0}, T_{1}\right)+O\left(\varepsilon^{2}\right) \\
& x\left(t-\tau_{2}, \varepsilon\right)=x_{0 \tau_{2}}\left(T_{0}, T_{1}\right)+\varepsilon x_{1 \tau_{2}}\left(T_{0}, T_{1}\right)+O\left(\varepsilon^{2}\right) \\
& v\left(t-\tau_{1}, \varepsilon\right)=v_{0 \tau_{1}}\left(T_{0}, T_{1}\right)+\varepsilon v_{1 \tau_{1}}\left(T_{0}, T_{1}\right)+O\left(\varepsilon^{2}\right) \\
& v\left(t-\tau_{3}, \varepsilon\right)=v_{0 \tau_{3}}\left(T_{0}, T_{1}\right)+\varepsilon v_{1 \tau_{3}}\left(T_{0}, T_{1}\right)+O\left(\varepsilon^{2}\right)
\end{aligned}
$$


The time derivatives of equations (1) and (2) can be written as follows in terms of two time scales:

$$
\frac{d}{d t}=D_{0}+\varepsilon D_{1}, \frac{d^{2}}{d t^{2}}=D_{0}^{2}+\varepsilon\left(2 D_{0} D_{1}\right), \quad D_{n}=\frac{\partial}{\partial \mathrm{T}_{n}}(n=0,1)
$$

We get the following result by inserting equations (3 to 5) into equations (1-2) and associating factors of equal power of $\varepsilon$ :

$O\left(\varepsilon^{0}\right)$ :

$$
\begin{aligned}
& \left(D_{0}^{2}+\omega_{s}^{2}\right) x_{0}=0 \\
& \left(D_{0}^{2}+\omega_{c}^{2}\right) v_{0}=0 \\
& O\left(\varepsilon^{1}\right):
\end{aligned}
$$

$$
\begin{aligned}
\left(D_{0}^{2}+\omega_{s}^{2}\right) x_{1}= & -2 D_{1} D_{0} x_{0}-\eta_{s} D_{0} x_{0}-\alpha x_{0}^{3}-\beta x_{0}\left(D_{0} x_{0}\right)^{2}-\gamma x_{0}^{2} D_{0} x_{0} \\
& +f \cos (\Omega t)+\gamma_{1} v_{0 \tau_{1}}^{2} \\
\left(D_{0}^{2}+\omega_{c}^{2}\right) v_{1}= & -2 D_{1} D_{0} v_{0}-\eta_{c} D_{0} v_{0}+\gamma_{2} x_{0 \tau_{2}} v_{0 \tau_{3}}
\end{aligned}
$$

Equations (6) have the following solutions:

$x_{0}\left(T_{0}, T_{1}\right)=A_{1}\left(T_{1}\right) \exp \left(i \omega_{s} T_{0}\right)+c c$.

$v_{0}\left(T_{0}, T_{1}\right)=A_{2}\left(T_{1}\right) \exp \left(i \omega_{c} T_{0}\right)+c c$.

Where, $A_{1}\left(T_{1}\right), A_{2}\left(T_{1}\right)$ are complex functions in $T_{1}, c c$. locate for the complex conjugate of the previous terms.

$v_{0 \tau_{1}}\left(T_{0}, T_{1}\right)=A_{2 \tau_{1}}\left(T_{1}\right) \exp \left(i \omega_{c}\left(T_{0}-\tau_{1}\right)\right)+c c$.

$v_{0 \tau_{1}}\left(T_{0}, T_{1}\right)=A_{2 \tau_{1}}\left(T_{1}\right) \exp \left(i \omega_{c}\left(T_{0}-\tau_{1}\right)\right)+c c$.

$v_{0 \tau_{3}}\left(T_{0}, T_{1}\right)=A_{2 \tau_{3}}\left(T_{1}\right) \exp \left(i \omega_{c}\left(T_{0}-\tau_{3}\right)\right)+c c$.

$A_{1 \tau_{2}}\left(T_{1}\right)=A_{1}\left(T_{1}-\varepsilon \tau_{2}\right) \cong A_{1}\left(T_{1}\right)-\varepsilon \tau_{2} A_{1}^{\prime}\left(T_{1}\right)$

$A_{2 \tau_{1}}\left(T_{1}\right)=A_{2}\left(T_{1}-\varepsilon \tau_{1}\right) \cong A_{2}\left(T_{1}\right)-\varepsilon \tau_{1} A_{2}^{\prime}\left(T_{1}\right)$

$A_{2 \tau_{3}}\left(T_{1}\right)=A_{2}\left(T_{1}-\varepsilon \tau_{3}\right) \cong A_{2}\left(T_{1}\right)-\varepsilon \tau_{3} A_{2}^{\prime}\left(T_{1}\right)$

Where ()$^{\prime}=\partial() / \partial T_{1}=\partial() / \partial(\varepsilon t)$

Substitute equations (8) interested in Equations (7), we obtain the following solutions:

$$
\begin{aligned}
x_{1}= & {\left[\frac{\alpha A_{1}^{3}-\beta \omega_{s}^{2} A_{1}^{3}+i \gamma \omega_{s} A_{1}^{3}}{8 \omega_{s}^{2}}\right] \exp \left(3 i \omega_{s} T_{0}\right)+\left[\frac{f}{2\left(\omega_{s}^{2}-\Omega^{2}\right)}\right] \exp \left(i \Omega T_{0}\right) } \\
& +\left[\frac{\gamma_{1} A_{2}^{2} \exp \left(-2 i \omega_{c} \tau_{1}\right)}{\omega_{s}^{2}-4 \omega_{c}^{2}}\right] \exp \left(2 i \omega_{c} T_{0}\right)+\left[\frac{\gamma_{1} A_{2} \overline{A_{2}}}{\omega_{s}^{2}}\right]+c c . \\
v_{1}= & {\left[\frac{\gamma_{2} A_{1} A_{2} \exp \left(-i\left(\omega_{s} \tau_{2}+\omega_{c} \tau_{3}\right)\right)}{\omega_{c}^{2}-\left(\omega_{s}+\omega_{c}\right)^{2}}\right] \exp \left(i\left(\omega_{s}+\omega_{c}\right) T_{0}\right) } \\
& +\left[\frac{\gamma_{2} A_{1} \overline{A_{2}} \exp \left(i\left(\omega_{c} \tau_{3}-\omega_{s} \tau_{2}\right)\right)}{\omega_{c}^{2}-\left(\omega_{s}-\omega_{c}\right)^{2}}\right] \exp \left(i\left(\omega_{s}-\omega_{c}\right) T_{0}\right)+c c .
\end{aligned}
$$


The complex conjugate function is indicated by the over bar wherever it appears. Before moving on to the following step of the solution, we necessity first determine the potential resonance cases at equations (9): primary resonance ( $\left.\Omega \cong \omega_{s}\right)$, internal resonance $\left(\omega_{s} \cong 2 \omega_{c}\right)$, and simultaneous resonance $\left(\Omega \cong \omega_{s}, \omega_{s} \cong 2 \omega_{c}\right)$, the worst of which. As a result, this case is explored by adding the two parameters $\sigma_{1}$ and $\sigma_{2}$ describing the closeness of the $\Omega$ and $\omega_{s}$ to $2 \omega_{c}$ as follows:

$\Omega \cong \omega_{s}+\varepsilon \sigma_{1}, \omega_{s} \cong 2 \omega_{c}+\varepsilon \sigma_{2}$

We get the following solvability conditions by plugging equations (10) into the smalldivisor and secular terms of equations (7):

$$
\begin{aligned}
2 i \omega_{s} D_{1} A_{1} & =\left[-i \omega_{s} \eta_{s} A_{1}+\left(-3 \alpha-\beta \omega_{s}^{2}-i \gamma \omega_{s}\right) A_{1}^{2} \overline{A_{1}}\right] \\
& +\left[\frac{f}{2}\right] \exp \left(i \sigma_{1} T_{1}\right)+\left[\gamma_{1} A_{2}^{2} \exp \left(-2 i \omega_{c} \tau_{1}\right)\right] \exp \left(-i \sigma_{2} T_{1}\right)
\end{aligned}
$$

$2 i \omega_{c} D_{1} A_{2}=-i \omega_{c} \eta_{c} A_{2}+\left[\gamma_{2} A_{1} \overline{A_{2}} \exp \left(i\left(\omega_{c} \tau_{3}-\omega_{s} \tau_{2}\right)\right)\right] \exp \left(i \sigma_{2} T_{1}\right)$

We rewrite $A_{1}\left(T_{1}\right)$ and $A_{2}\left(T_{1}\right)$ in polar form to analyze equations (11), as follows:

$A_{n}=\frac{1}{2} a_{n} \exp \left(i \beta_{n}\right), \quad n=1,2$

Where $a_{1}$ and $a_{2}$ are the system's steady-state amplitudes, $\beta_{1}$ and $\beta_{2}$ are the phases of the two movements, respectively. We get the following set of first-order differential equations by applying equations (12) to equations (11) and sorting out the real and imaginary components.

$$
\begin{aligned}
& \dot{a}_{1}=-\frac{\eta_{s}}{2} a_{1}-\frac{\gamma}{8} a_{1}^{3}+\left[\frac{f}{2 \omega_{s}}\right] \sin \left(\theta_{1}\right)-\left[\frac{\gamma_{1}}{4 \omega_{s}} a_{2}^{2} \cos \left(2 \omega_{c} \tau_{1}\right)\right] \sin \left(\theta_{2}\right) \\
& -\left[\frac{\gamma_{1}}{4 \omega_{s}} a_{2}^{2} \sin \left(2 \omega_{c} \tau_{1}\right)\right] \cos \left(\theta_{2}\right) \\
& a_{1} \dot{\beta}_{1}=\left(\frac{3 \alpha}{8 \omega_{s}}+\frac{\beta \omega_{s}}{8}\right) a_{1}^{3}+\left[-\frac{f}{2 \omega_{s}}\right] \cos \left(\theta_{1}\right)+\left[-\frac{\gamma_{1}}{4 \omega_{s}} a_{2}^{2} \cos \left(2 \omega_{c} \tau_{1}\right)\right] \cos \left(\theta_{2}\right) \\
& +\left[\frac{\gamma_{1}}{4 \omega_{s}} a_{2}^{2} \sin \left(2 \omega_{c} \tau_{1}\right)\right] \sin \left(\theta_{2}\right) \\
& \dot{a}_{2}=-\frac{\eta_{c}}{2} a_{2}+\left[\frac{\gamma_{2}}{4 \omega_{c}} a_{1} a_{2} \cos \left(\omega_{c} \tau_{3}-\omega_{s} \tau_{2}\right)\right] \sin \left(\theta_{2}\right)+\left[\frac{\gamma_{2}}{4 \omega_{c}} a_{1} a_{2} \sin \left(\omega_{c} \tau_{3}-\omega_{s} \tau_{2}\right)\right] \cos \left(\theta_{2}\right) \\
& a_{2} \dot{\beta}_{2}=\left[-\frac{\gamma_{2}}{4 \omega_{c}} a_{1} a_{2} \cos \left(\omega_{c} \tau_{3}-\omega_{s} \tau_{2}\right)\right] \cos \left(\theta_{2}\right)+\left[\frac{\gamma_{2}}{4 \omega_{c}} a_{1} a_{2} \sin \left(\omega_{c} \tau_{3}-\omega_{s} \tau_{2}\right)\right] \sin \left(\theta_{2}\right)
\end{aligned}
$$


Where $\theta_{1}=\sigma_{1} T_{1}-\beta_{1}, \theta_{2}=\sigma_{2} T_{1}+\beta_{1}-2 \beta_{2}$. Equations $(13,14)$ forms the system amplitude-phase modulating equations.

$$
\begin{aligned}
& a_{1} \dot{\theta}_{1}=a_{1} \sigma_{1}-\left(\frac{3 \alpha}{8 \omega_{s}}+\frac{\beta \omega_{s}}{8}\right) a_{1}^{3}+\left[\frac{f}{2 \omega_{s}}\right] \cos \left(\theta_{1}\right)+\left[\frac{\gamma_{1}}{4 \omega_{s}} a_{2}^{2} \cos \left(2 \omega_{c} \tau_{1}\right)\right] \cos \left(\theta_{2}\right) \\
& -\left[\frac{\gamma_{1}}{4 \omega_{s}} a_{2}^{2} \sin \left(2 \omega_{c} \tau_{1}\right)\right] \sin \left(\theta_{2}\right) \\
& a_{2} \dot{\theta}_{2}=a_{2}\left(\sigma_{2}+\sigma_{1}\right)-a_{2} \dot{\theta}_{1}+\left[\frac{\gamma_{2}}{2 \omega_{c}} a_{1} a_{2} \cos \left(\omega_{c} \tau_{3}-\omega_{s} \tau_{2}\right)\right] \cos \left(\theta_{2}\right)-\left[\frac{\gamma_{2}}{2 \omega_{c}} a_{1} a_{2} \sin \left(\omega_{c} \tau_{3}-\omega_{s} \tau_{2}\right)\right] \sin \left(\theta_{2}\right)
\end{aligned}
$$

\section{Study of Stability}

The fluctuations in amplitudes and phases of a constantly excited system are zero at steady state. Equations $(13,14)$ can be used to determine the algebraic equations that control the steady state vibrations of the considered structure as a function of the environment $\dot{a}_{1}=\dot{a}_{2}=\dot{\theta}_{1}=\dot{\theta}_{2}=0$, yielding

$$
\begin{aligned}
& \frac{\eta_{s}}{2} a_{1}+\frac{\gamma}{8} a_{1}^{3}=\left[\frac{f}{2 \omega_{s}}\right] \sin \left(\theta_{1}\right)-\left[\frac{\gamma_{1}}{4 \omega_{s}} a_{2}^{2} \cos \left(2 \omega_{c} \tau_{1}\right)\right] \sin \left(\theta_{2}\right) \\
& -\left[\frac{\gamma_{1}}{4 \omega_{s}} a_{2}^{2} \sin \left(2 \omega_{c} \tau_{1}\right)\right] \cos \left(\theta_{2}\right) \\
& a_{1} \sigma_{1}-\left(\frac{3 \alpha}{8 \omega_{s}}+\frac{\beta \omega_{s}}{8}\right) a_{1}^{3}=-\left[\frac{f}{2 \omega_{s}}\right] \cos \left(\theta_{1}\right)-\left[\frac{\gamma_{1}}{4 \omega_{s}} a_{2}^{2} \cos \left(2 \omega_{c} \tau_{1}\right)\right] \cos \left(\theta_{2}\right) \\
& +\left[\frac{\gamma_{1}}{4 \omega_{s}} a_{2}^{2} \sin \left(2 \omega_{c} \tau_{1}\right)\right] \sin \left(\theta_{2}\right) \\
& \frac{\eta_{c}}{2} a_{2}=\left[\frac{\gamma_{2}}{4 \omega_{c}} a_{1} a_{2} \cos \left(\omega_{c} \tau_{3}-\omega_{s} \tau_{2}\right)\right] \sin \left(\theta_{2}\right)+\left[\frac{\gamma_{2}}{4 \omega_{c}} a_{1} a_{2} \sin \left(\omega_{c} \tau_{3}-\omega_{s} \tau_{2}\right)\right] \cos \left(\theta_{2}\right) \\
& \frac{a_{2}}{2}\left(\sigma_{2}+\sigma_{1}\right)=\left[-\frac{\gamma_{2}}{4 \omega_{c}} a_{1} a_{2} \cos \left(\omega_{c} \tau_{3}-\omega_{s} \tau_{2}\right)\right] \cos \left(\theta_{2}\right)+\left[\frac{\gamma_{2}}{4 \omega_{c}} a_{1} a_{2} \sin \left(\omega_{c} \tau_{3}-\omega_{s} \tau_{2}\right)\right] \sin \left(\theta_{2}\right)
\end{aligned}
$$

We have the following cases based on equations (15) and (16):

$$
\text { (i) } a_{1} \neq 0, a_{2}=0 \text { (ii) } a_{2} \neq 0, a_{1}=0 \text { and (iii) } a_{1} \neq 0, a_{2} \neq 0
$$

To endorse the useful case (iii), square equation (15), then add the squared results together, and repeat for equation (16). Finally, we include the following frequency response equations: 


$$
\begin{aligned}
& \left(\frac{\eta_{s}}{2} a_{1}+\frac{\gamma}{8} a_{1}^{3}\right)^{2}+\left(a_{1} \sigma_{1}-\left(\frac{3 \alpha}{8 \omega_{s}}+\frac{\beta \omega_{s}}{8}\right) a_{1}^{3}-\frac{\gamma_{1} \omega_{c}}{2 \omega_{s} \gamma_{2} a_{1}} a_{2}^{2}\left(\sigma_{2}+\sigma_{1}\right)\right)^{2}=\frac{f^{2}}{4 \omega_{s}^{2}}+\left(\frac{\gamma_{1}}{\omega_{s}} a_{2}^{2}\right)^{2} \\
& \frac{\eta_{c}^{2}}{4}+\frac{1}{4}\left(\sigma_{2}+\sigma_{1}\right)^{2}+\frac{\eta_{c}}{2}\left(\sigma_{2}+\sigma_{1}\right)=\frac{\gamma_{2}^{2}}{8 \omega_{c}^{2}} a_{1}^{2}
\end{aligned}
$$

To perform stability criteria, we assume $a_{10}, a_{20}, \theta_{10}$ and $\theta_{20}$ are the solutions of equations (15) and (16), and to observe the performance of small perturbations $a_{11}, a_{21}, \theta_{11}$ and $\theta_{21}$ from the steady state solution $a_{10}, a_{20}, \theta_{10}$ and $\theta_{20}$, we let

$$
\begin{array}{ll}
a_{1}=a_{11}+a_{10}, \quad a_{2}=a_{21}+a_{20}, & \theta_{1}=\theta_{11}+\theta_{10}, \theta_{2}=\theta_{21}+\theta_{10} \\
\dot{a}_{1}=\dot{a}_{11}, \quad \dot{a}_{2}=\dot{a}_{21}, \quad \dot{\theta}_{1}=\dot{\theta}_{11}, & \dot{\theta}_{2}=\dot{\theta}_{21}
\end{array}
$$

Substituting equation (19) into equations $(13,14)$ and extending for small parameters $a_{11}, a_{21}, \theta_{11}$ and $\theta_{21}$ continuing only in linear terms. We compact the linear structure that is equivalent to equations $(13,14)$ at the equilibrium point $\left(a_{10}, a_{20}, \theta_{10}, \theta_{20}\right)$ :

$$
\left[\begin{array}{c}
\dot{a}_{11} \\
\dot{\theta}_{11} \\
\dot{a}_{21} \\
\dot{\theta}_{21}
\end{array}\right]=\left[\begin{array}{cccc}
r_{11} & r_{12} & r_{13} & r_{14} \\
r_{21} & r_{22} & r_{23} & r_{24} \\
r_{31} & r_{32} & r_{33} & r_{34} \\
r_{41} & r_{42} & r_{43} & r_{44}
\end{array}\right]\left[\begin{array}{c}
a_{11} \\
\theta_{11} \\
a_{21} \\
\theta_{21}
\end{array}\right]
$$

The method Jacobian matrix and its coefficients are prearranged in the appendix wherever the over matrix is. The following eigenvalue equations can be obtained:

$$
\left|\begin{array}{cccc}
r_{11}-\lambda & r_{12} & r_{13} & r_{14} \\
r_{21} & r_{22}-\lambda & r_{23} & r_{24} \\
r_{31} & r_{32} & r_{33}-\lambda & r_{34} \\
r_{41} & r_{42} & r_{43} & r_{44}-\lambda
\end{array}\right|=0
$$

Expanding the determinant at (21), we get

$$
\lambda^{4}+\zeta_{1} \lambda^{3}+\zeta_{2} \lambda^{2}+\zeta_{3} \lambda+\zeta_{4}=0
$$

Where $\lambda$ is the eigenvalue of the matrix, $\zeta_{1}, \zeta_{2}, \zeta_{3}$ and $\zeta_{4}$ are the coefficients of equation (22) known in the appendix. The required and proper conditions for a stable solution, as determined by the Routh-Hurwitz criterion, are:

$$
\zeta_{1}>0, \quad \zeta_{1} \zeta_{2}-\zeta_{3}>0, \quad \zeta_{3}\left(\zeta_{1} \zeta_{2}-\zeta_{3}\right)-\zeta_{1}^{2} \zeta_{4}>0, \quad \zeta_{4}>0
$$




\section{Results and discussions}

The data is represented graphically. The effects of the parameters $\tau_{1}, \tau_{2}, \tau_{3}, \sigma_{1}, \gamma_{1}, \gamma_{2}$ and $f$ on the chief structure's and controller's dynamics have investigated. Unless otherwise mentioned, Figs. $2-22$ have plotted using the next device parameters: $\eta_{s}=0.02, \omega_{s}=1, \alpha=0.894, \beta=0.0001, \gamma=0.0001, \Omega \cong \omega_{s}, \gamma_{1}=0.2$, $\sigma_{1}=0, \sigma_{2}=0, \eta_{c}=0.0001,2 \omega_{c}=\omega_{s}, \gamma_{2}=0.1$, and $f=0.05$. Fig. 2 depicts amplitudedelay $\tau_{1}$ response curves for $\tau_{2}=\tau_{3}=0$, while Fig. 3 portrays amplitude-delay $\tau_{2}$ response curves for $\tau_{1}=\tau_{2}=0$ and Fig. 4 displays response curves of amplitude-delay $\tau_{3}$ for $\tau_{1}=\tau_{2}=0$. As seen in the figures below, the amplitude-response delay curves are periodic in time delays, meaning that evaluating the amplitude-response delay curves in one period is appropriate. The amplitude-delay response curve is stable for small time delays, but when time delays reach critical levels, the solution takes on an unpredictable shape that happens on a regular basis. The time interval in which the amplitude-delay response curves have a stable solution is known as the "vibration suppression area". Solid lines denote stable solutions, dotted lines represent unstable solutions, and asterisks represent the difference between stable and unstable solutions.
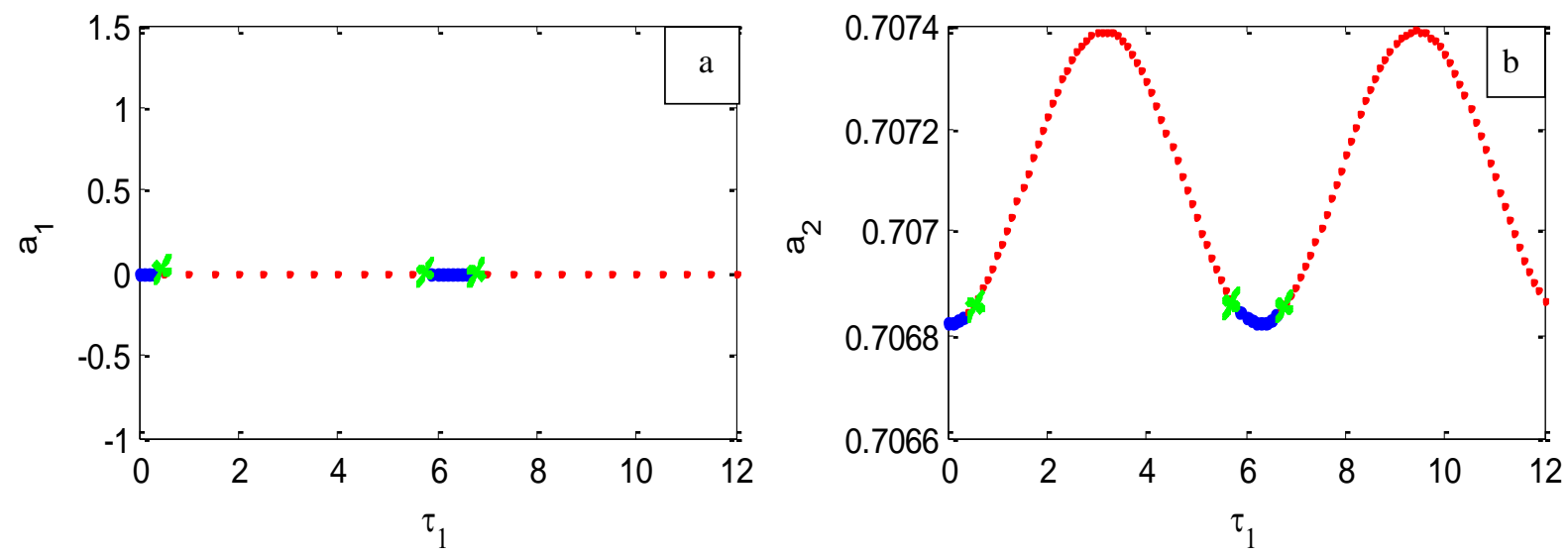

Fig. 2. Curves of amplitude-delay $\tau_{1}$ response, (a) key system and (b) controller
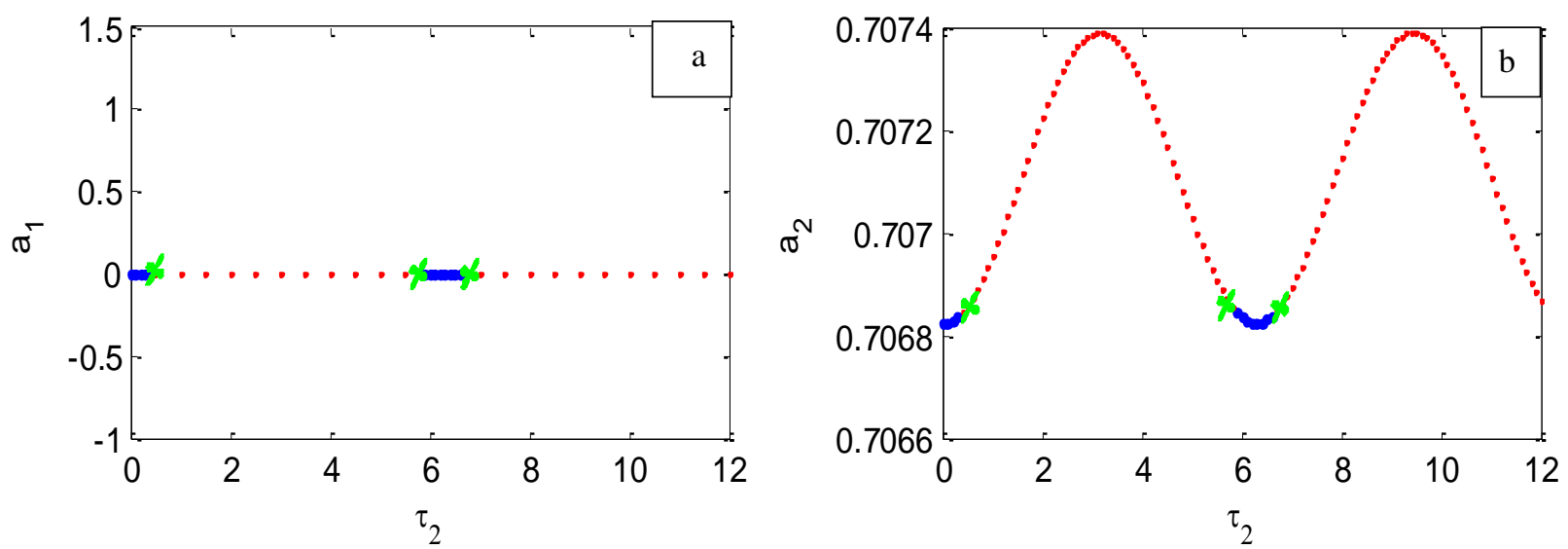

Fig. 3. Curves of amplitude-delay $\tau_{2}$ response, (a) basic system and (b) controller 

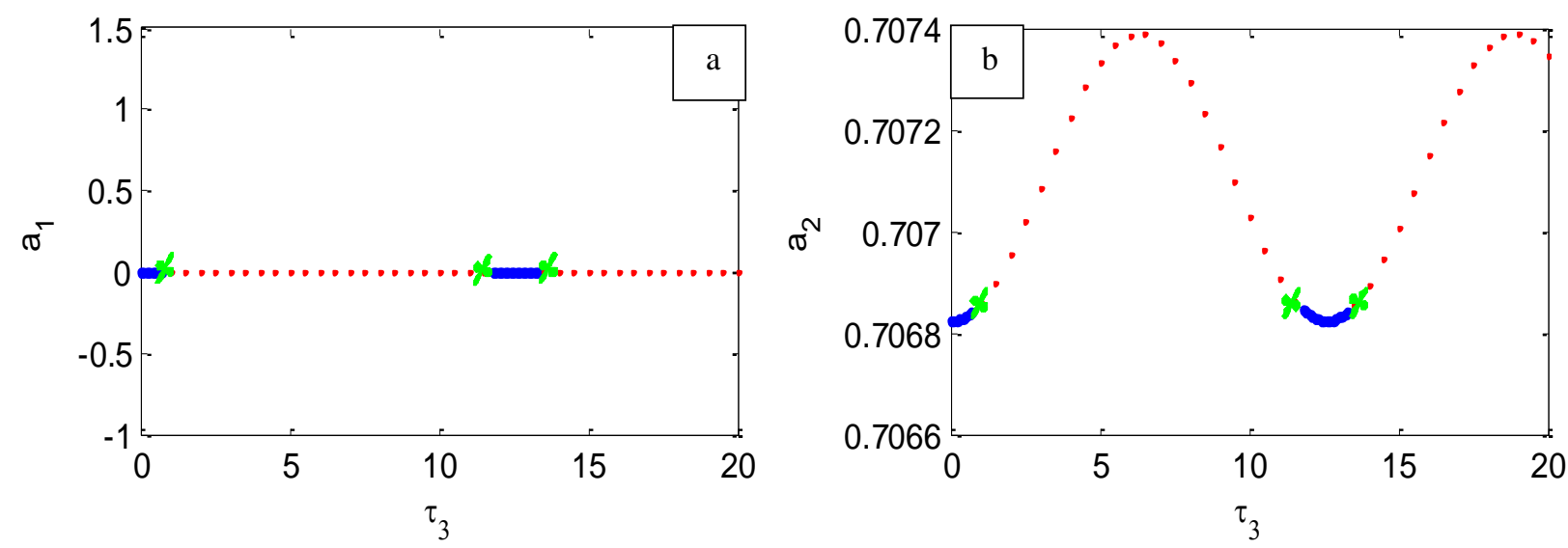

Fig. 4. Curves of amplitude-delay $\tau_{3}$ response, (a) chief system and (b) controller

Without any time delay, the frequency response curves for the main system and controller (closed loop case) are displayed in Fig. 5. As $\sigma_{1}$ increases from left to right, we can see that the main system amplitude follows the blue curve's course and behaves in the same way without control until it reaches point A. At this stage, the controller is automatically activated, causing the amplitude of the main system to change and follow the direction of the other blue curve. The main system amplitude decreases as $\sigma_{1}$ increases until it reaches the minimum value at $\sigma_{1}=0$ (optimum operating point), then it rises again on the same curve until it reaches point $\mathrm{B}$, where the controller is automatically deactivated, forcing the main system amplitude to leave the curve's path and return to the blue curve's path, allowing it to behave in the same manner without the controller. As seen in this diagram, the minimum main system amplitude occurs at $\sigma_{1}=0$ and the most suitable operating range for the controller is to operate within approximately $\sigma_{1}= \pm 0.02$.
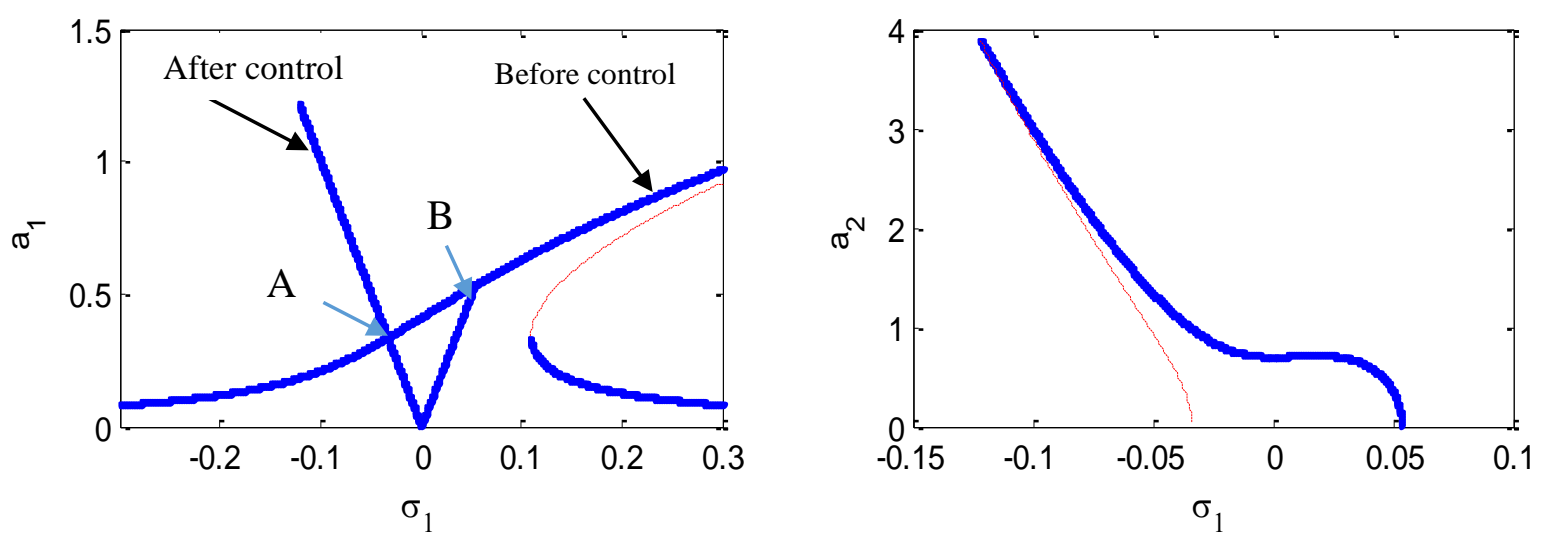

Fig. 5 Frequency response curves of the main system and controller without time delays at $\sigma_{2}=0$.

Figs. 6 and 7 show how time delays influence the controller's frequency-response curves. As $\tau_{1}$ or/and $\tau_{2}$ rises but within the range of "the vibration reduction domain," as seen in Fig. 6, the controller amplitude $a_{2}$ decreases for frequency of excitation $\Omega$ 
smaller than the main system natural frequency $\omega_{1}$ (i.e. for negative values of $\sigma_{1}$ ), and the controller's overload event stated in ref [11]. As shown in Fig. 7, as the value $\tau_{3}$ increases, the controller can exhibit a complex unstable motion, so it's best to keep it as close to zero as possible.
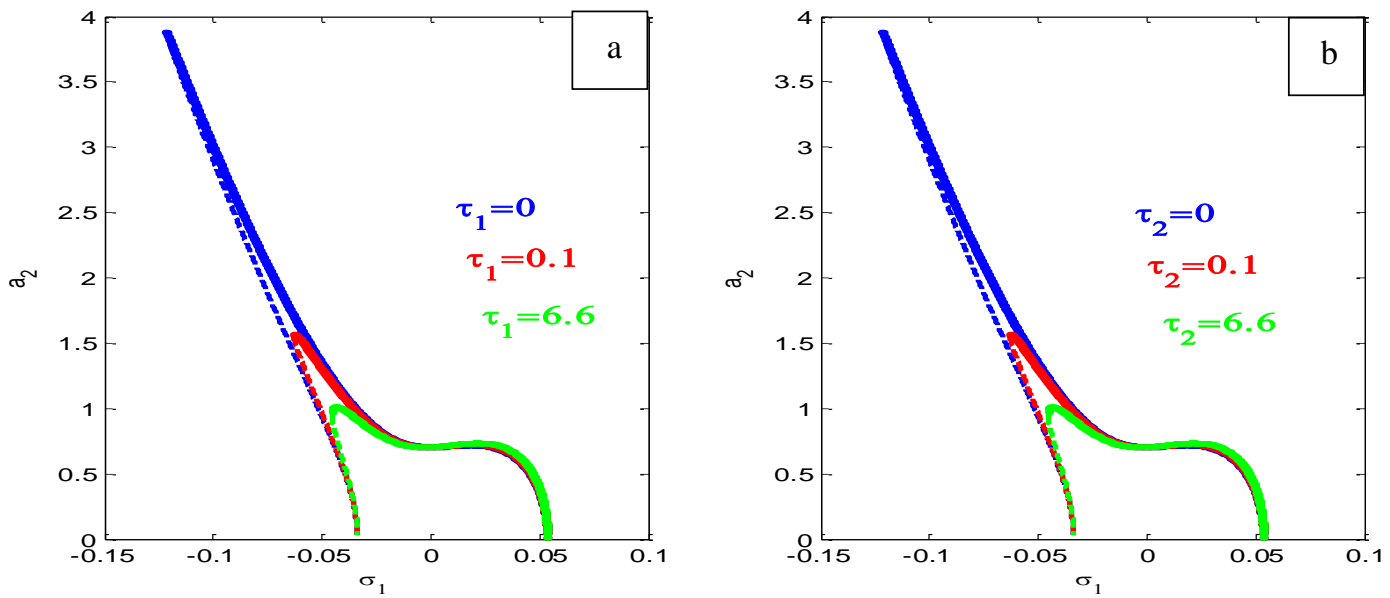

Fig. 6. The outcome of time delays on the frequency-response curves of the controller::

(a) time delay $\tau_{1}$ when $\tau_{2}=\tau_{3}=0$ and (b) time delay $\tau_{2}$ when $\tau_{1}=\tau_{3}=0$.
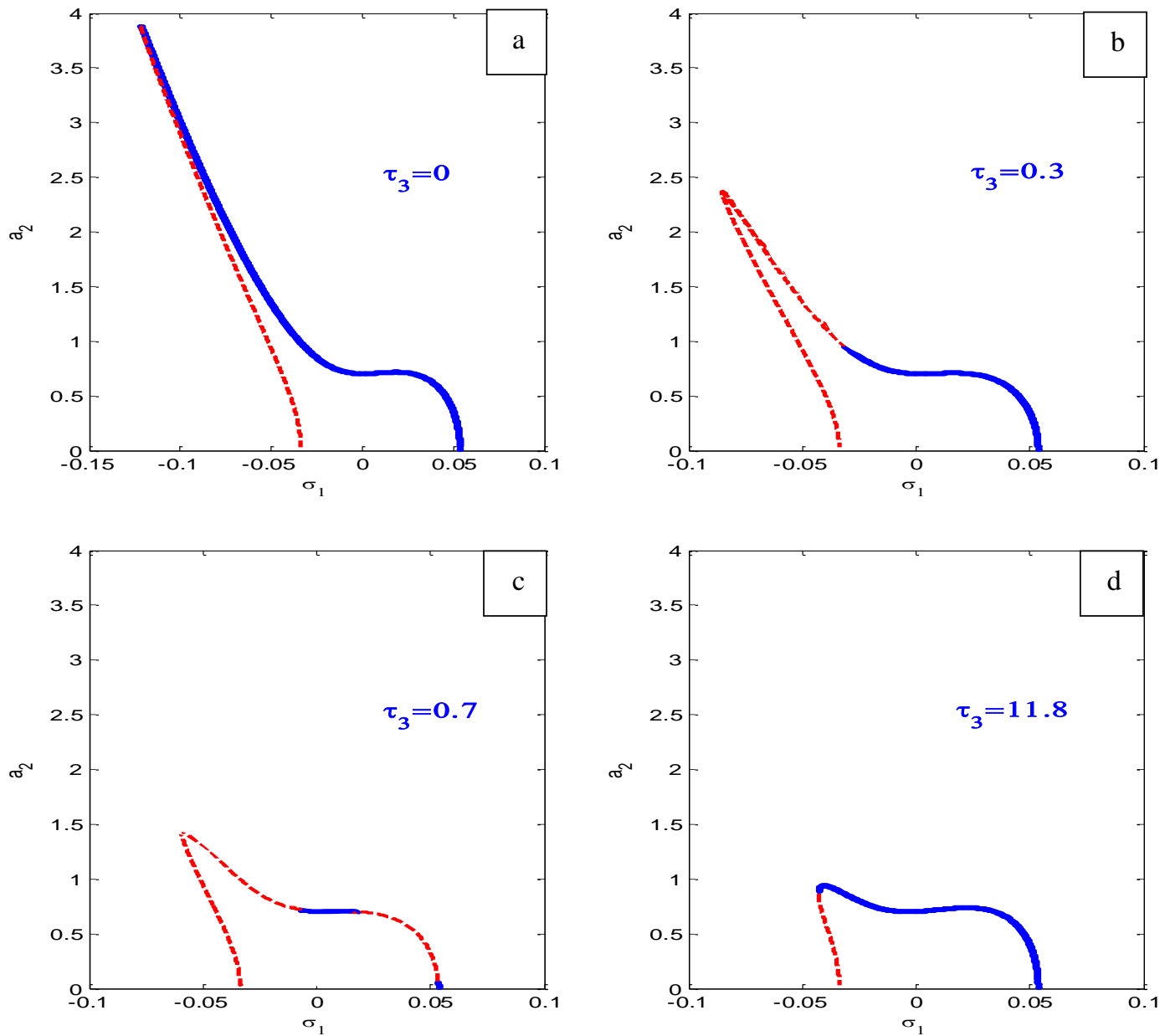
Fig. 7. Impact of the time delay $\tau_{3}$ on the frequency-response curves of the controller

$$
\text { when } \tau_{1}=\tau_{2}=0 \text {. }
$$

Fig. 8 shows the effects of varying the control signal gain $\gamma_{1}$ on the frequency response curves for both the main system and the controller at $\tau_{1}=0.3, \tau_{2}=0.3, \tau_{3}=0.7$. As shown in Eq. (18), the amplitude of the main machine is clearly independent of $\gamma_{1}$, while the amplitude of the controller is a monotonic decreasing function in $\gamma_{1}$
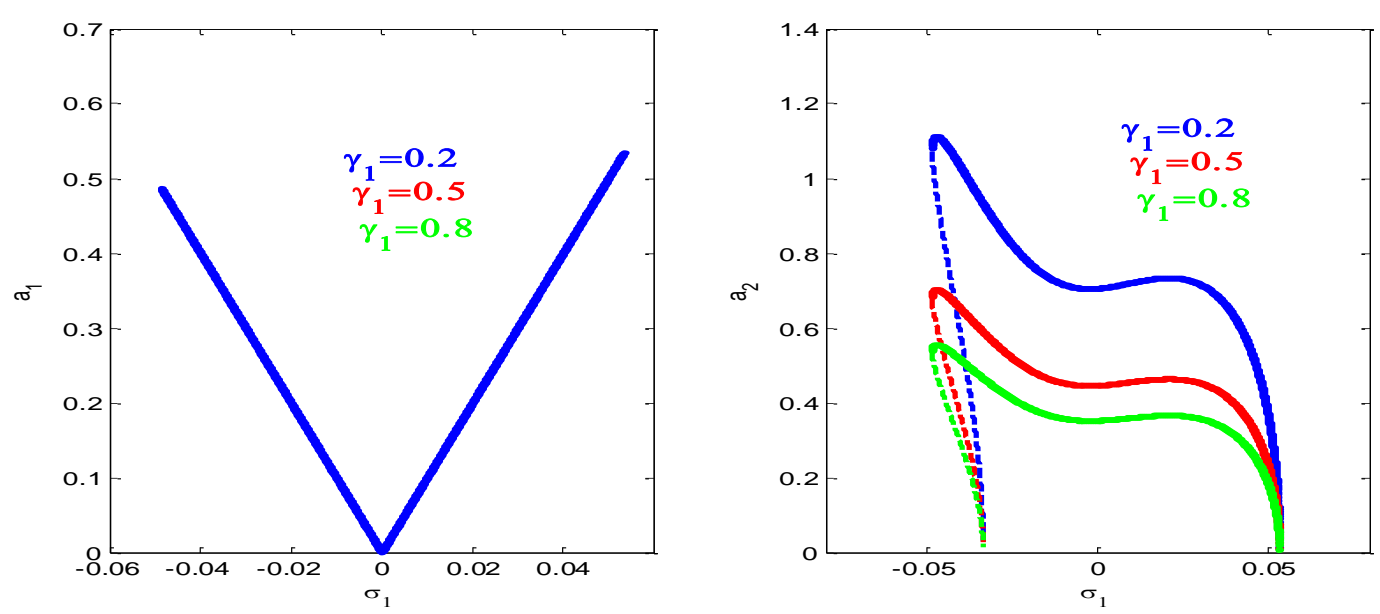

Fig. 8. The frequency response diagrams of the chief system and controller for various values of $\gamma_{1}$ when $\tau_{1}=0.3, \tau_{2}=0.3, \tau_{3}=0.7$.

Fig. 9 shows the effects of adjusting the feedback signal gain $\gamma_{2}$ on the frequency response curves of the main system and controller at $\tau_{1}=0.3, \tau_{2}=0.3, \tau_{3}=0.7$. The bandwidth of the minimum amplitudes of the main system, as well as the bandwidth of the controller operation, grows $\gamma_{2}$ as the bandwidth of the main system's minimum amplitudes grows.
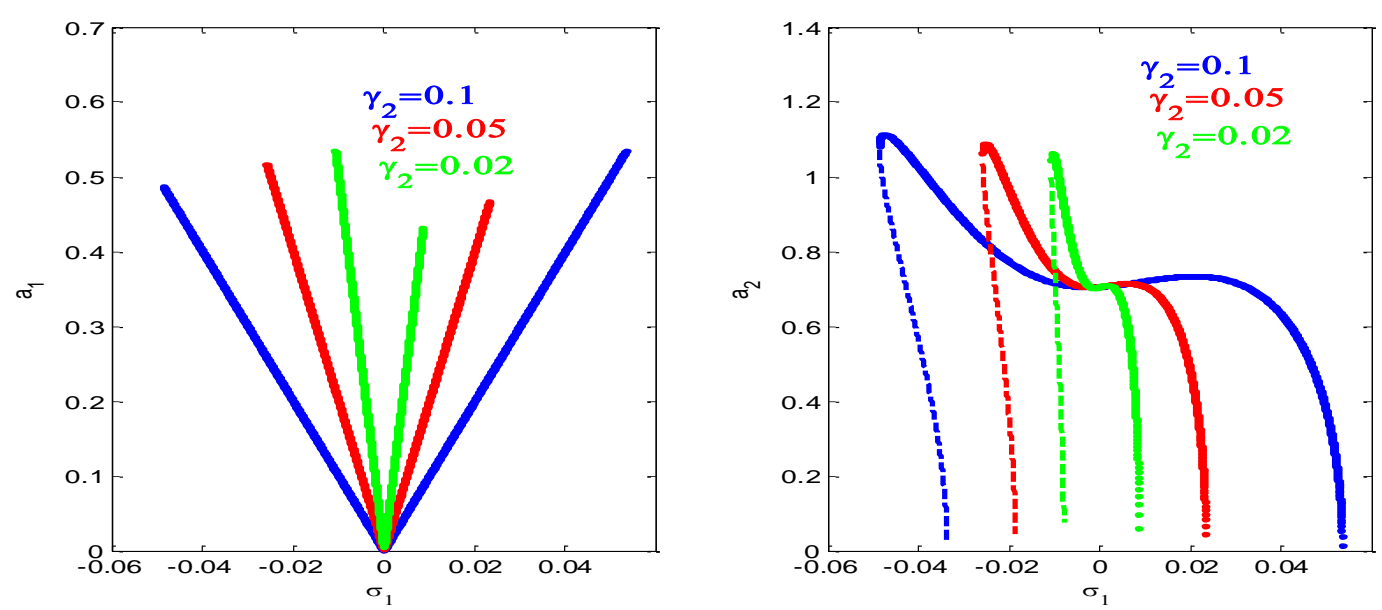

Fig. 9. The frequency response diagrams of the main system and controller for various values of $\gamma_{2}$ when $\tau_{1}=0.3, \tau_{2}=0.3, \tau_{3}=0.7$. 
The force-response curves of the main machine and the controller (controller in action) for time delays $\tau_{1}=\tau_{2}=\tau_{3}=0$ are shown in Figs. 10a and 10b. As shown in Fig. 10a, the main system's amplitude increases as the excitation force increases until a critical value is reached, at which point the main system's amplitude saturates and all excess energy due to the excitation force is channeled to the controller, as shown in Fig. 10b. This supports the well-known saturation phenomenon, which states that "when the response of a directly excited system reaches a critical value, it does not differ with excitation amplitude." Figs. 10c and 10d display the effect of time delays on forceresponse curves. The chosen time delays values are $\tau_{1}=0.3, \tau_{2}=0.3, \tau_{3}=0.7$ in this case. The saturation phenomenon is confirmed in Figs. 10c and 10d, but it should be noted that as time delays increase, the stable regions of force-response curves shrink.
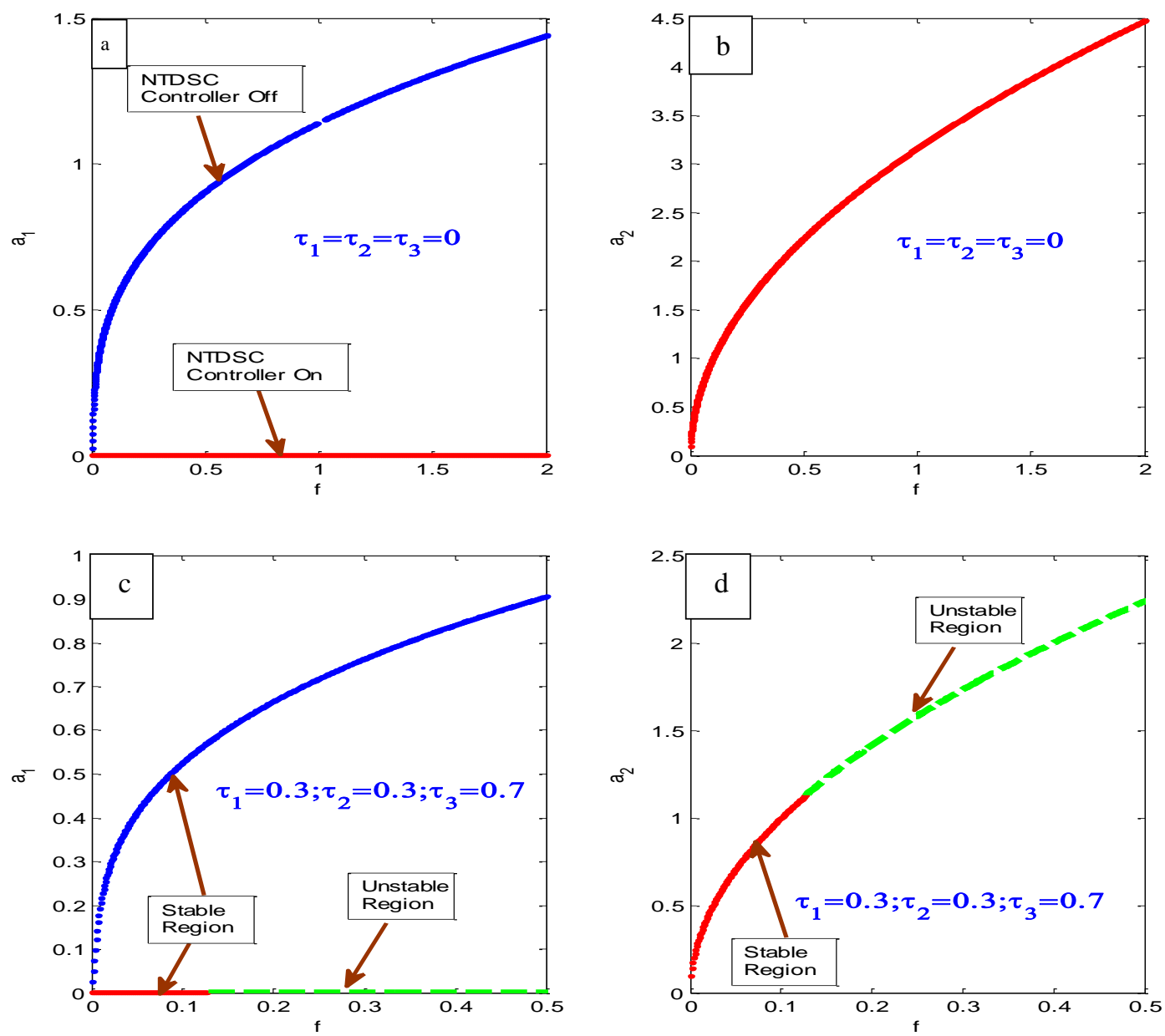

Fig. 10. Force-amplitude response curves of: $(a, b)$ when controller is turned on and off without time delays and (c, d) when controller is turned on and off on with time delays $\tau_{1}=0.3, \tau_{2}=0.3, \tau_{3}=0.7$. 


\section{Numerical simulations}

Eqs. (1) and (2) were numerically integrated using MATLAB software to verify the effects of the perturbation tests, and the outcomes for stable equilibrium solutions were exposed in small circles in Figs. 11. The variation of $a_{1}$ and $a_{2}$ against $\sigma_{1}$ for $\tau_{1}=\tau_{2}=\tau_{3}=0$ is depicted in Fig. 11

Stable regions,

Unstable regions, and

o Numerical integration are depicted in this diagram. In addition, as shown in Fig. 11, all analytical predictions agree very well with the numerical simulation. Fig. 12 depicts the device without any acted control at simultaneous resonance $\left(\Omega \cong \omega_{s}, \omega_{s} \cong 2 \omega_{c}\right)$. Figs. 13 and 14 show when the NTDSC is turned off and when it is turned on. These figures depict the time when the NTDSC was enabled at $t=89$ seconds. Figs. 15-20 display the time responses of the structure and controller for different values of time delays using the same previously selected parameter as in the previous section. With initial conditions $x(0)=0, \dot{x}(0)=0, v(0)=0.5, \dot{v}(0)=0$ for $\tau_{1}=\tau_{2}=\tau_{3}=0$, Fig. 15 depicts stable actions for the structure and controller. With the same initial conditions but at $\tau_{1}=0.3, \tau_{2}=0.3, \tau_{3}=0.7$, the response curve behavior of the scheme and the controller appears at Fig.16. In addition, Fig. 17-20 Repeat with different values of $\tau_{1}=\tau_{2}=0.3, \tau_{3}=0.7 \quad, \quad \tau_{1}=\tau_{2}=0.1, \tau_{3}=11.8 \quad, \quad \tau_{1}=\tau_{2}=6.6, \tau_{3}=11.8 \quad$ and $\tau_{1}=6.6, \tau_{2}=\tau_{3}=0$.
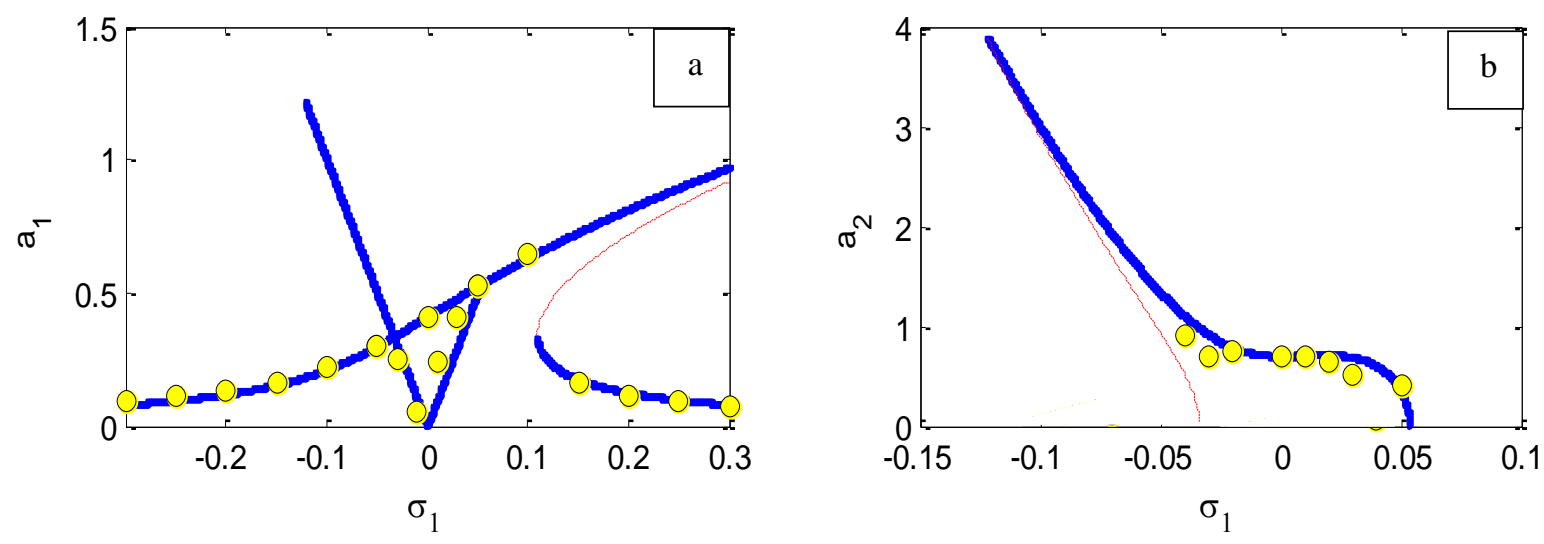

Fig. 11. The frequency-response curves of: (a) the main framework and (b) the controller.
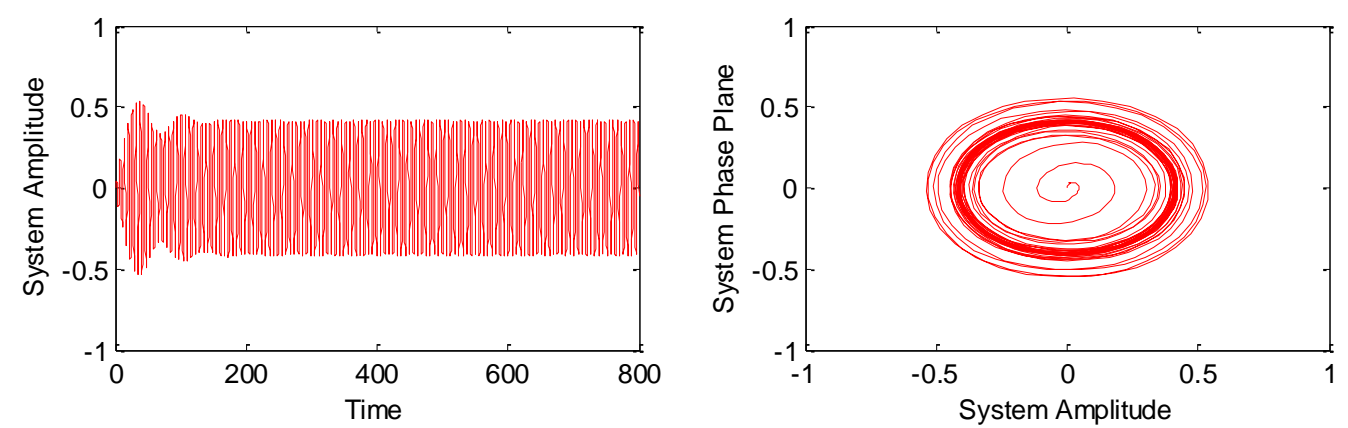
Fig. 12. Response of the framework with no controller at simultaneous resonance $\left(\Omega \cong \omega_{s}, \omega_{s} \cong 2 \omega_{c}\right)$.

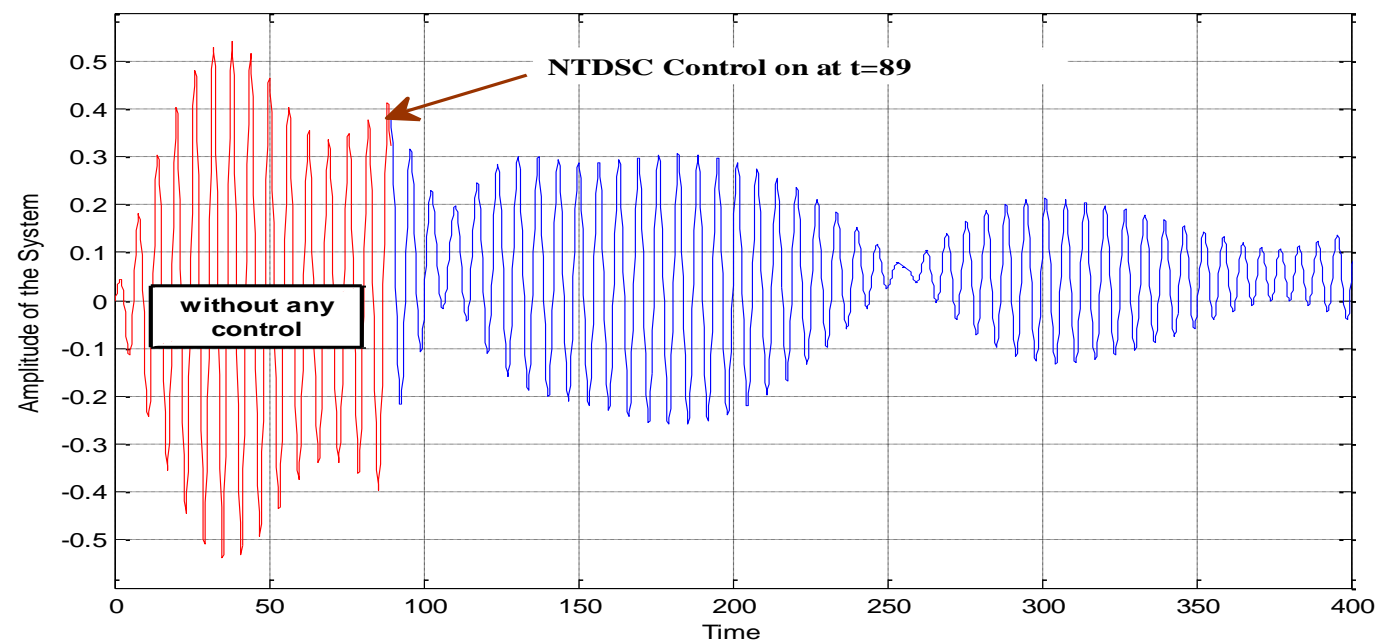

Fig. 13. Curve appears that the system with no controller and at $t=89$ the NTDSC is in action.

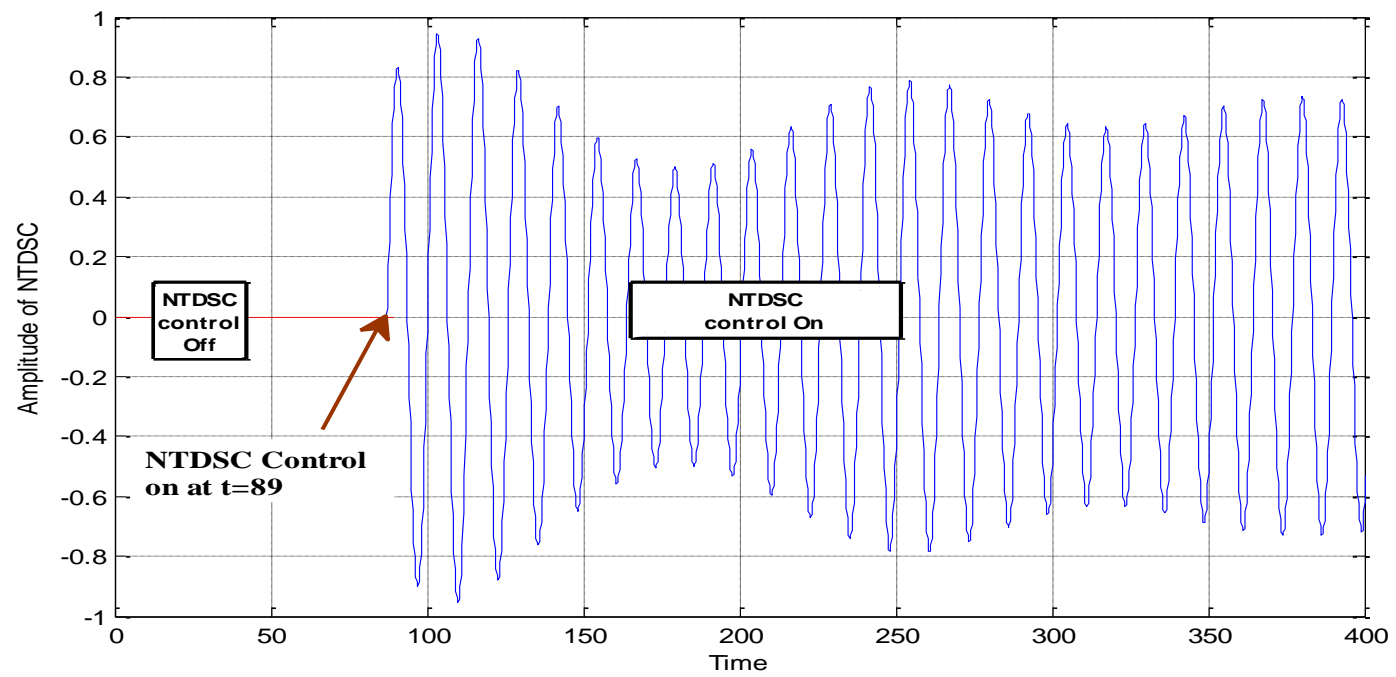

Fig. 14. Curve appears that the controller when it off and is on at $t=89 \mathrm{sec}$.
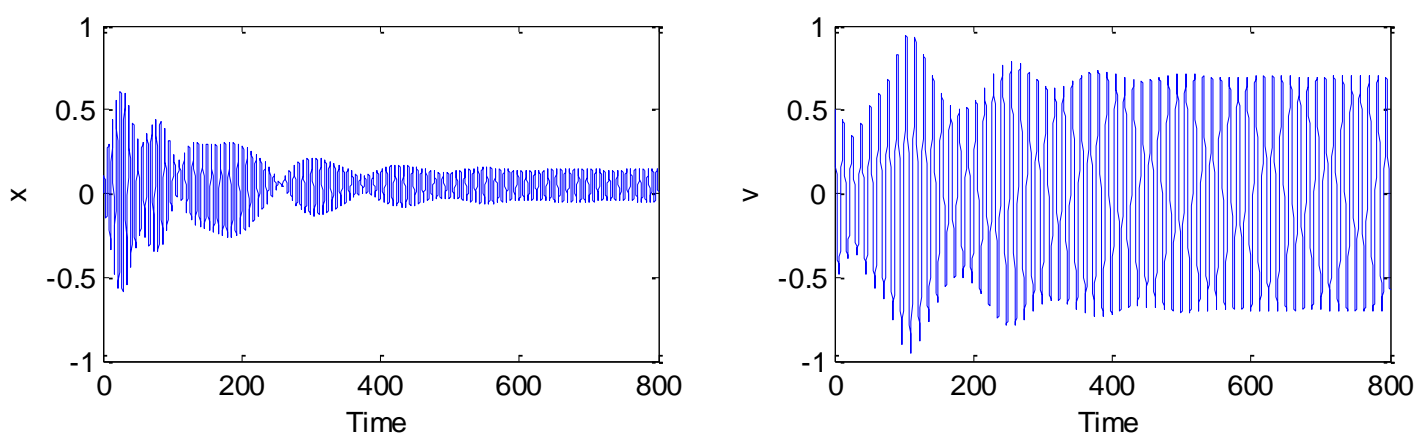

Fig. 15. Numerical simulations of the main system's and controller's time responses when $x(0)=0, \dot{x}(0)=0, v(0)=0.5, v^{\circ}(0)=0, \tau_{1}=0, \tau_{2}=0, \tau_{3}=0$ 

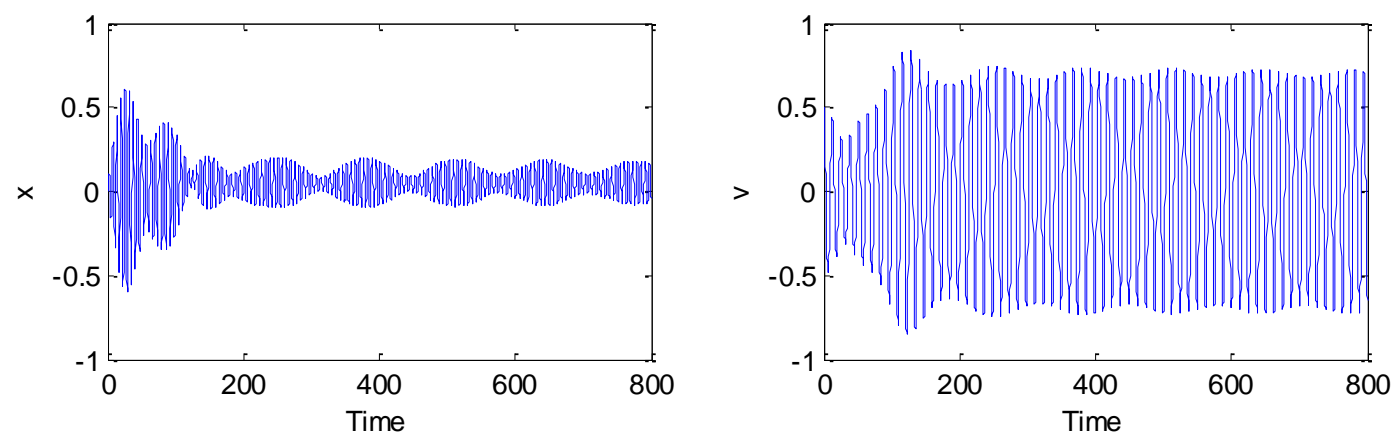

Fig. 16. Numerical simulations of the main system's and controller's time responses when $x(0)=0, \dot{x}(0)=0, v(0)=0.5, \dot{v}(0)=0, \tau_{1}=0.3, \tau_{2}=0.3, \tau_{3}=0.3$
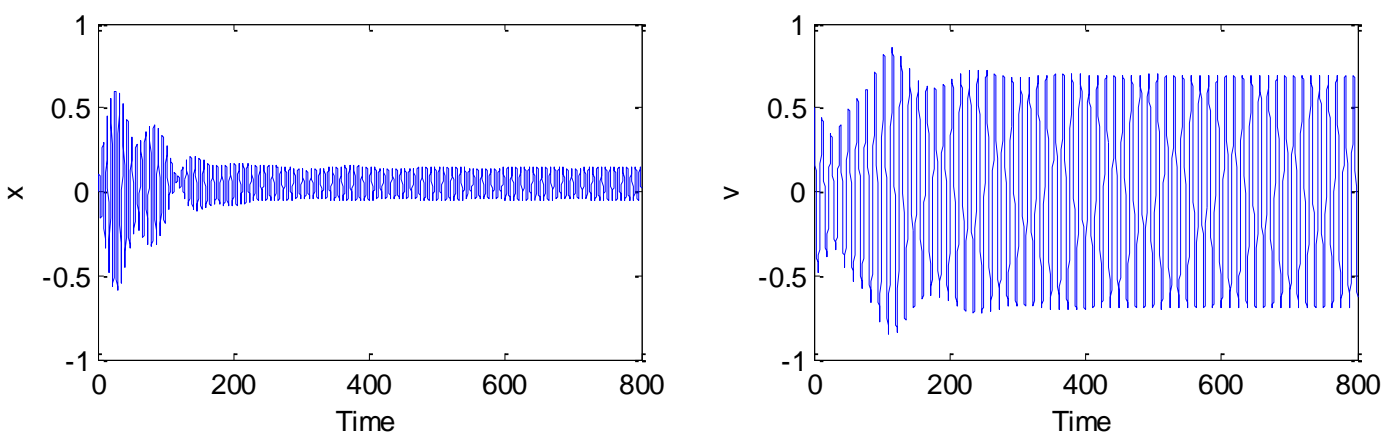

Fig. 17. Numerical simulations of the main system's and controller's time responses when $x(0)=0, \dot{x}(0)=0, v(0)=0.5, \dot{v}(0)=0, \tau_{1}=0.3, \tau_{2}=0.3, \tau_{3}=0.7$
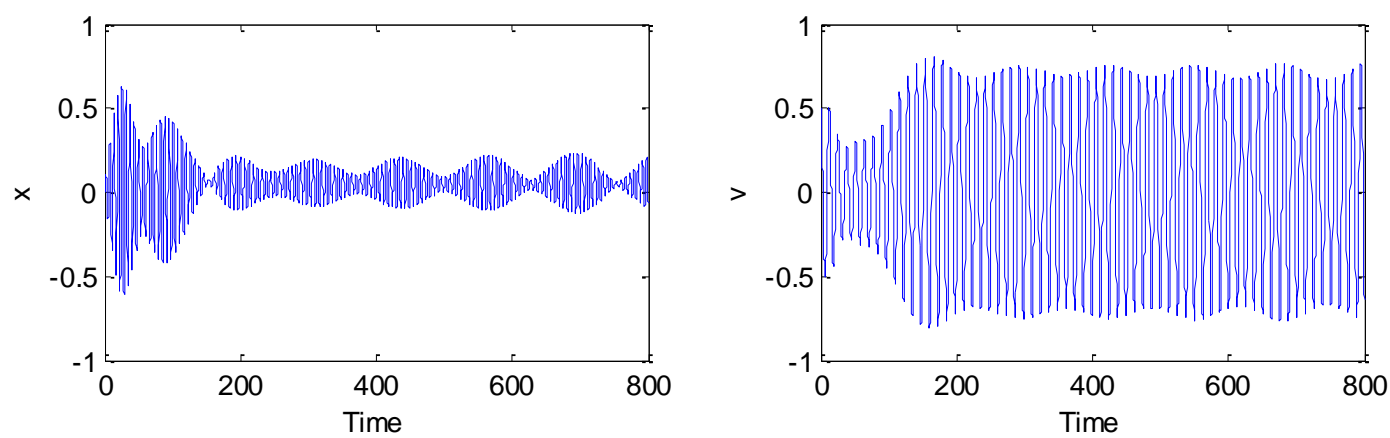

Fig. 18. Numerical simulations of the main system's and controller's time responses when $x(0)=0, \dot{x}(0)=0, v(0)=0.5, \dot{v}(0)=0, \tau_{1}=0.1, \tau_{2}=0.1, \tau_{3}=11.8$
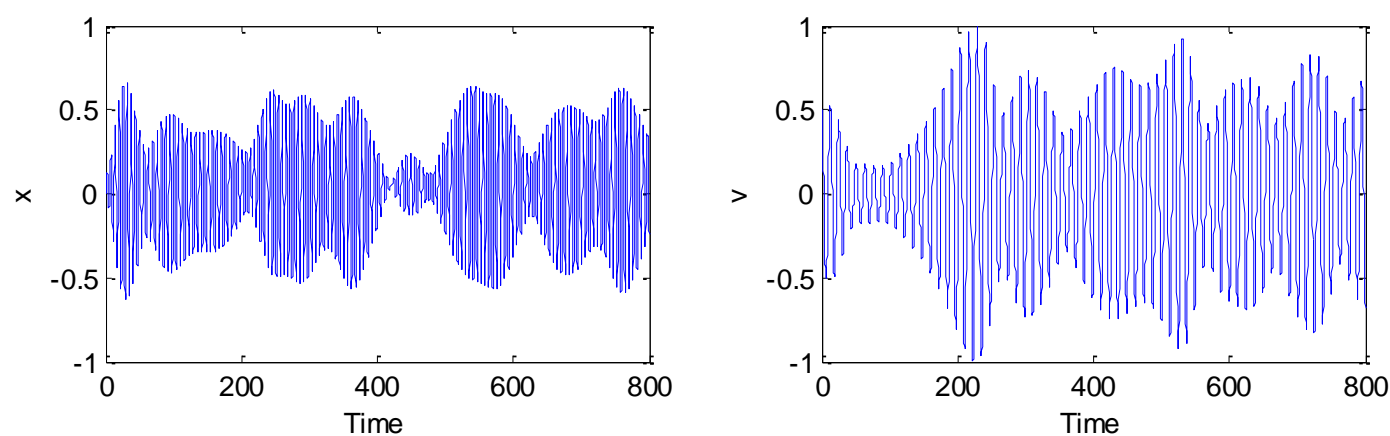

Fig. 19. Numerical simulations of the main system's and controller's time responses when $x(0)=0, \dot{x}(0)=0, v(0)=0.5, \dot{v}(0)=0, \tau_{1}=6.6, \tau_{2}=6.6, \tau_{3}=11.8$ 

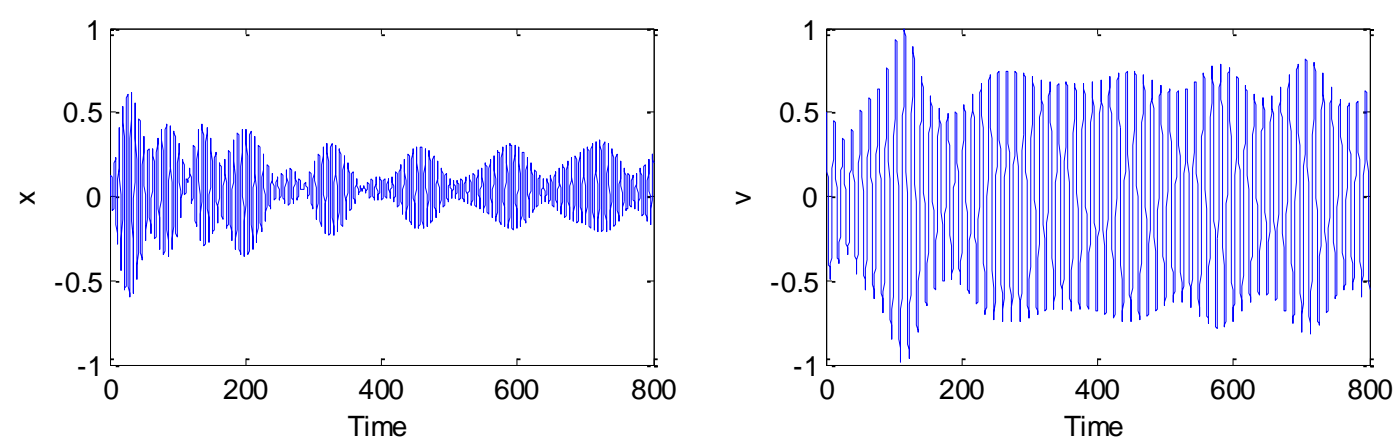

Fig. 20. Numerical simulations of the main system's and controller's time responses when $x(0)=0, \dot{x}(0)=0, v(0)=0.5, \dot{v}(0)=0, \tau_{1}=6.6, \tau_{2}=0, \tau_{3}=0$.

\subsection{Relationship between analytical and numerical solutions}

For the resonance case ( $\Omega \cong \omega_{s}, \omega_{s} \cong 2 \omega_{c}$ ), the series of equations (13) and (14) defines modulation of the amplitudes $a_{1}, a_{2}$ and the modified phases $\theta_{1}, \theta_{2}$. In Figs. 21 and 22, the solutions of equations (13) and (14) for various values of the function parameters are graphically shown. The modulation of the amplitudes for the generalized coordinates $x$ and $v$ is shown by the dashed lines. The continuous lines, on the other hand, reflect the time history of vibrations obtained numerically as solutions of the original Eqs. (1) and (2). (2). The graphed solutions were obtained by using the following values for some of the parameters:

$\eta_{s}=0.02, \omega_{s}=1, \alpha=0.894, \beta=0.0001, \gamma=0.0001, \Omega \cong \omega_{s}, \gamma_{1}=0.2$, $\sigma_{1}=0, \sigma_{2}=0, \eta_{c}=0.0001,2 \omega_{c}=\omega_{s}, \gamma_{2}=0.1$ and $f=0.05$. The values of time delays $\tau_{1}=\tau_{2}=\tau_{3}=0$ are plotted in Fig. 21, while the values of $\tau_{1}=\tau_{2}=0.3, \tau_{3}=0.7$ are plotted in Fig. 22. The intensive energy exchange between modes of vibration and energy transfer between the main device and controller can be seen in Figs. 21 and 22.
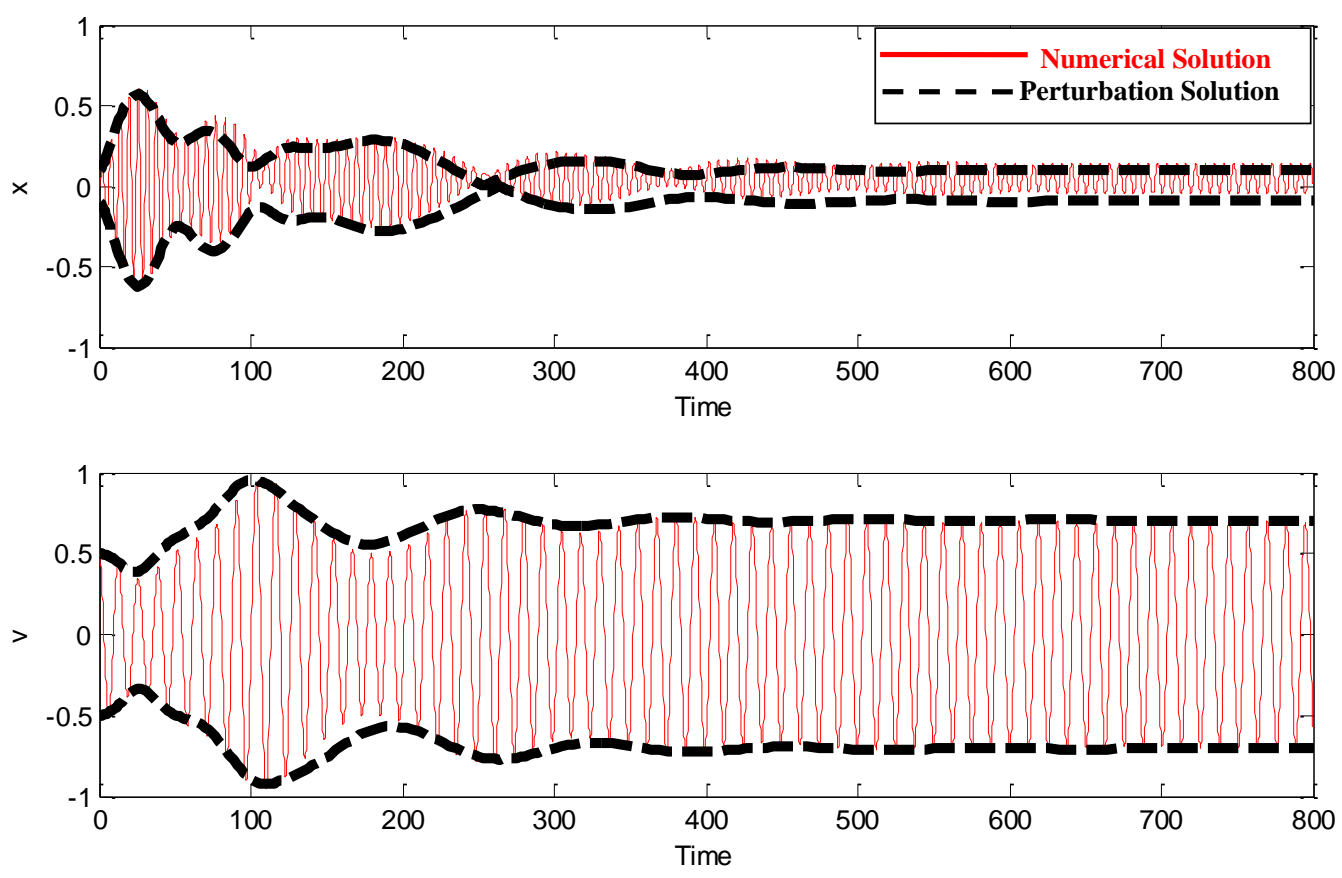
Fig. 21. Comparison among perturbation investigation and numerical simulation of the main system and the controller when

$$
\tau_{1}=0, \tau_{2}=0, \tau_{3}=0, x(0)=0, \dot{x}(0)=0, v(0)=0.5, \dot{v}(0)=0 \text {. }
$$
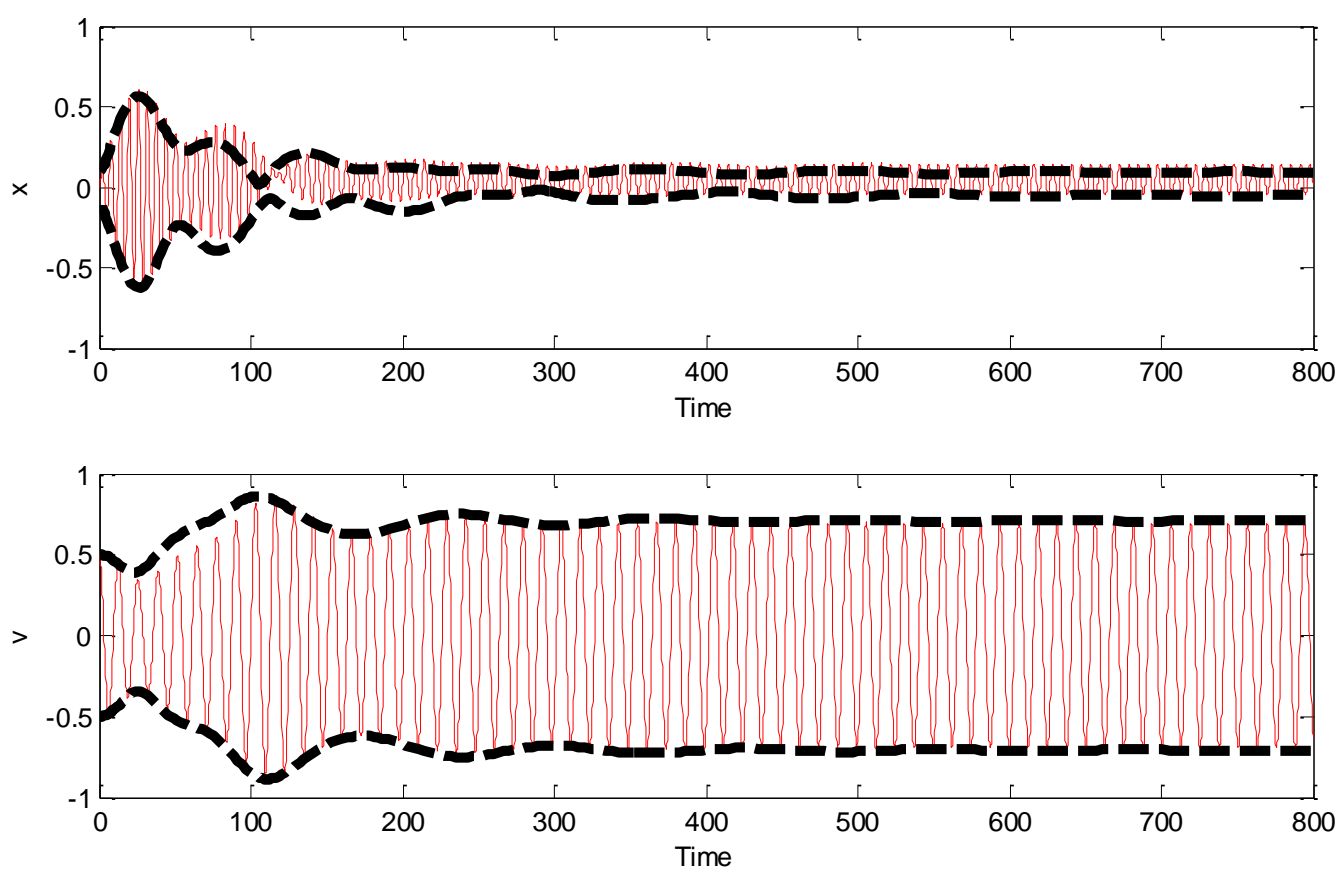

Fig. 22. Comparison among perturbation investigation and numerical simulation of the main system and the controller when

$$
\tau_{1}=0.3, \tau_{2}=0.3, \tau_{3}=0.7, x(0)=0, \dot{x}(0)=0, v(0)=0.5, \dot{v}(0)=0
$$

\subsection{Comparison between our paper and recent published papers with same model}

The authors of Ref. [7] proposed using a saturation-based active absorber to inhibit a nonlinear plant vibration caused by a primary external excitation. They discovered that the control scheme has a large suppression bandwidth once the absorber's frequency is properly tuned. In addition, the authors of Ref. [11] investigated a saturation-based controller for nonlinear beam vibration suppression, but time delays are inherent in any active control device. Authors investigated a harmonically excited dynamical system with new NIPPF, IRC, and PPF controllers in Ref. [16]. They used perturbation efficiency at primary and 1:1 internal resonance to study the device analytically with NIPPF. With zero initial conditions, they may reduce the vibration amplitude by using NIPPF, PPF, and IRC to approximately $84.87 \%, 82.24 \%$ and $46.71 \%$, respectively, from the value of the uncontrolled device. In addition, the authors of Ref. [24] used a delayed saturation controller to inhibit vibration in a stainless-steel beam. They demonstrated that the saturation control range can be altered by altering the time delays, which can be thought of as regulating parameters for suppressing the vibration of the regulated beam. Furthermore, the authors of Ref. [25] used delayed feedback control and saturation control to inhibit dynamical system vibration. They discovered that in a dynamical system, time delay is very significant. It can be used as a control parameter to not only stabilize or inhibit the primary system's vibration, but also to cause the 
system to move in an unstable manner. In addition, the authors of Ref. [28] investigated the effects of delayed feedback control on a nonlinear vibration absorber device. They discovered that at such delay values, the vibration can be suppressed. So, in this paper, we added three time delays $\tau_{1}, \tau_{2}$ and $\tau_{3}$ to the equations of the model in Ref. [16] to illustrate their effects on the main system and controller responses. We discovered that by shrinking or expanding the controller's frequency bandwidth, the time delays add new control keys to the system's answer, overcoming the problem of controller overload danger that occurs at frequencies of excitation lower than the main system's natural frequency. The results of the simulations revealed that all of the analytical solutions' estimates are in excellent agreement with the numerical simulation.

\section{Conclusions}

For the simultaneously resonance case $\left(\Omega \cong \omega_{s}, \omega_{s} \cong 2 \omega_{c}\right)$ of a dynamical device associated with a vertical cantilever beam simulated to an aircraft tail, the NTDSC method was investigated. The perturbation method is used to derive four first order differential equations that govern the time evolution of the system and controller's amplitudes and phases. The bifurcation analysis is then used to check the closed loop system's stability as well as the performance of the control rule. The investigations revealed that:

1. In some dynamical systems, time delays can be used as a control parameter to reduce vibration.

2. Unique values of time delays may be used to adjust the saturation control's range. The saturation control's effective frequency bandwidth may be reduced or increased. It is dependent on the values of the time delays as shown in Figs. 6 and 7.

3. For excitation frequencies lower than the main system's natural frequency, the controller's amplitude can be minimized by using specific values of time delays.

4. As shown in Figs 13 and 14, the time when the controller NTDSC is in action is registered at $\mathrm{t}=89 \mathrm{sec}$.

5. Despite time delays, it can be used to stabilize unstable systems; it can also be used to generate chaotic motions from stable systems by specifying arbitrary time delays, as shown in Figs. 15-20.

Data availability Data will be made available on reasonable request

\section{Declarations}

Conflict of interest The authors declare that they have no conflict of interest concerning the publication of this manuscript. 


\section{References}

[1] Oueini SS, Nayfeh AH, Pratt JR. A nonlinear vibration absorber for flexible structures. Nonlinear Dynamics, 15, 259-282 (1998).

[2] Pai PF, Wen B, Naser AS, Schulz MJ. Structural vibration control using PZT patches and non-linear phenomena. J. Sound Vibration, 215(2), 273-296 (1998).

[3] Oueini SS, Nayfeh AH. Single-mode control of a cantilever beam under principal parametric excitation. Journal of Sound and Vibration, 224(1), 33-47 (1999).

[4] Pai PF, Schulz MJ. A refined nonlinear vibration absorber. International Journal of Mechanical Science, 42, 537-560 (2000).

[5] Pai PF, Rommel B, Schulz MJ. Non-linear vibration absorbers using higher order internal resonances. Journal of Sound and Vibration, 234, 799-817 (2000).

[6] Ashour ON, Nayfeh AH. Adaptive control of flexible structures using a nonlinear vibration absorber. Nonlinear Dynamics, 28, 309-322 (2002).

[7] Jun L, Hongxing H and Rongying S. Saturation-based active absorber for a nonlinear plant to a principal external excitation. Mechanical Systems and Signal Processing, 21, 1489-1498 (2007).

[8] Jun L, Xiaobin L and Hongxing H. Active nonlinear saturation-based control for suppressing the free vibration of a self-excited plant. Communications in Nonlinear Science and Numerical Simulation, 15, 1071-1079 (2010).

[9] $\mathrm{Xu} \mathrm{J}$, Chung KW and Zhao YY. Delayed saturation controller for vibration suppression in a stainless-steel beam. Nonlinear Dynamics, 62, 177-193 (2010).

[10] Mitura A, Warminski J, Bochenski M and Kazmir T. Optimization of NSC controller in two-dimensional parameters space. ENOC 2011, 24-29 July, Rome, Italy (2011).

[11] Warminski J, Bochenski M, Jarzyna W, Filipek P and Augustyniak M. Active suppression of nonlinear composite beam vibrations by selected control algorithms. Communications in Nonlinear Science and Numerical Simulation, 16 (5), 2237-2248 (2011).

[12] Saeed NA, El-Ganini WA, Eissa M. Nonlinear time delay saturation-based controller for suppression of nonlinear beam vibrations. Applied Mathematical Modelling, 37, 8846-8864 (2013).

[13] Hamed YS, and Elagan SK. On the Vibration Behavior Study of a Nonlinear Flexible Composite Beam Under Excitation Forces via Nonlinear Active Vibration Controller. International Journal of Basic \& Applied Sciences, 13 (1), 9-18 (2013). 
[14] Hamed YS and Amer YA. Nonlinear saturation controller for vibration supersession of a nonlinear composite beam. Journal of Mechanical Science and Technology, 28 (8), 2987-3002 (2014).

[15] Kamel M, Kandil A, El-Ganaini WA and Eissa M. Active vibration control of a nonlinear magnetic levitation system via Nonlinear Saturation Controller (NSC). Nonlinear Dynamics, 77, 605-619 (2014).

[16] Omidi E and Mahmoodi SN. Sensitivity analysis of the Nonlinear Integral Positive Position Feedback and Integral Resonant controllers on vibration suppression of nonlinear oscillatory systems. Communications in Nonlinear Science and Numerical Simulation, 22, 149-166 (2015)

[17] Abdelhafez HM and Nassar ME. Suppression of vibrations of a forced and selfexcited nonlinear beam using positive position feedback controller PPF. Br. J. Math. Comput. Sci, 17 (4) 1-19 (2016).

[18] Abdelhafez HM and Nassar ME. Effects of time delay on active vibration control of a forced and self-excited nonlinear beam, Nonlinear Dyn., 86 137-151 (2016).

[19] Warminski J and Latalski J. Saturation control for rotating thin-walled composite beam structures, Procedia Eng. 144 713-720 (2016).

[20] Warminski, J., Cartmell, M.P., Mitura, A., Bochenski, M.: Active vibration control of a nonlinear beam with self-and external excitations. Shock Vib. 20 1033-1047 (2013).

[21] Jian X., Chen Y., and Chung KW. An improved time-delay saturation controller for suppression of nonlinear beam vibration, Nonlinear Dyn. 82 1691-1707 (2015).

[22] Dimitrios, H.-V., William, S.L. (eds.): In: Handbook of Networked and Embedded Control Systems, Control engineering. Birkhüser (2005).

[23]El-Ganaini, W.A., Kandil, A., Eissa, M., Kamel, M.: Effects of delayed time active controller on the vibration of a nonlinear magnetic levitation system subjected to multi excitations. J. Vib Control 22, 1257-1275 (2014).

[24]Xu, J., Chung, K.W., Zhao, Y.Y.: Delayed saturation controller for vibration suppression in stainless-steel beam. Nonlinear Dyn. 62, 177-193 (2010).

[25]Zhao, Y.Y., Xu, J.: Using the delayed feedback control and saturation control to suppress the vibration of dynamical system. Nonlinear Dyn. 67, 735-753 (2012). 
[26] Coppola, G., Liu, K.: Time-delayed position feedback control for a unique active vibration isolator. Struct. Control Heal. Monit. 19, 646-666 (2012).

[27]Zhao, Y.Y., Xu, J.: Effects of delayed feedback control on nonlinear vibration absorber system. J. Sound Vib. 308, 212- 230 (2007).

[28] Amer, Y.A., Soleman, S.M.: The time delayed feedback control to suppress the vibration of the auto parametric dynamical system. Sci. Res. Essays. 10, 489-500 (2015).

[29] Elnaggar, A.M., Khalil, K.M.: The response of nonlinear controlled system under an external excitation via time delay state feedback. J. King Saudi Univ. Eng. Sci. 28, 75-83 (2016).

[30] Phohomsiri, P., Udwadia, F.E., von Bremen, H.F.: Time delayed positive velocity feedback control design for active control of structures. J. Eng. Mech. 6, 690-703 (2006).

[31] Gao, X., Chen, Q.: Active Vibration Control for a Bilinear System with Nonlinear Velocity Time-delayed Feedback, vol. 3. World Congress on Engineering, London (2013).

[32]Zhang J., Lu M., Shen T., Liu L., Bo Y., Sliding mode control for a class of nonlinear multi-agent system with time delay and uncertainties, IEEE Trans. Ind. Electron. 65 (1) 865-875 (2017).

[33] Mondal J., and Chatterjee S., Controlling self-excited vibration of a nonlinear beam by nonlinear resonant velocity feedback with time-delay. Int. J. of Nonlinear Mech., 131103684 (2021).

[34] Nayfeh A. H.: Perturbation Methods. Wiley, New York (1973).

[35] Nayfeh A. H., Mook, D.: Nonlinear Oscillations. Wiley, New York (1995).

\section{Appendix}

$$
\begin{aligned}
& r_{11}=\left[-\frac{\eta_{s}}{2}-\frac{3 \gamma}{8} a_{10}^{2}\right], r_{12}=\left[\frac{f}{2 \omega_{s}} \cos \left(\theta_{10}\right)\right], \\
& r_{13}=\left[-\frac{\gamma_{1}}{2 \omega_{s}} a_{20} \cos \left(2 \omega_{c} \tau_{1}\right) \sin \left(\theta_{20}\right)-\frac{\gamma_{1}}{2 \omega_{s}} a_{20} \sin \left(2 \omega_{c} \tau_{1}\right) \cos \left(\theta_{20}\right)\right],
\end{aligned}
$$




$$
\begin{aligned}
& r_{14}=\left[-\frac{\gamma_{1}}{4 \omega_{s}} a_{20}^{2} \cos \left(2 \omega_{c} \tau_{1}\right) \cos \left(\theta_{20}\right)+\frac{\gamma_{1}}{4 \omega_{s}} a_{20}^{2} \sin \left(2 \omega_{c} \tau_{1}\right) \sin \left(\theta_{20}\right)\right], \\
& r_{21}=\left[\frac{\sigma_{1}}{a_{10}}-\left(\frac{9 \alpha}{8 \omega_{s}}+\frac{3 \beta \omega_{s}}{8}\right) a_{10}\right], r_{22}=\left[-\frac{f}{2 a_{10} \omega_{s}} \sin \left(\theta_{10}\right)\right] \text {, } \\
& r_{23}=\left[\frac{\gamma_{1} a_{20}}{2 a_{10} \omega_{s}} \cos \left(2 \omega_{c} \tau_{1}\right) \cos \left(\theta_{20}\right)-\frac{\gamma_{1} a_{20}}{2 a_{10} \omega_{s}} \sin \left(2 \omega_{c} \tau_{1}\right) \sin \left(\theta_{20}\right)\right] \text {, } \\
& r_{24}=\left[-\frac{\gamma_{1} a_{20}^{2}}{4 a_{10} \omega_{s}} \cos \left(2 \omega_{c} \tau_{1}\right) \sin \left(\theta_{20}\right)-\frac{\gamma_{1} a_{20}^{2}}{4 a_{10} \omega_{s}} \sin \left(2 \omega_{c} \tau_{1}\right) \cos \left(\theta_{20}\right)\right] \text {, } \\
& r_{31}=\left[\frac{\gamma_{2}}{4 \omega_{c}} a_{20} \cos \left(\omega_{c} \tau_{3}-\omega_{s} \tau_{2}\right) \sin \left(\theta_{20}\right)+\frac{\gamma_{2}}{4 \omega_{c}} a_{20} \sin \left(\omega_{c} \tau_{3}-\omega_{s} \tau_{2}\right) \cos \left(\theta_{20}\right)\right], r_{32}=0 \text {, } \\
& r_{33}=\left[-\frac{\eta_{c}}{2}+\frac{\gamma_{2}}{4 \omega_{c}} a_{10} \cos \left(\omega_{c} \tau_{3}-\omega_{s} \tau_{2}\right) \sin \left(\theta_{20}\right)+\frac{\gamma_{2}}{4 \omega_{c}} a_{10} \sin \left(\omega_{c} \tau_{3}-\omega_{s} \tau_{2}\right) \cos \left(\theta_{20}\right)\right] \text {, } \\
& r_{34}=\left[\frac{\gamma_{2}}{4 \omega_{c}} a_{10} a_{20} \cos \left(\omega_{c} \tau_{3}-\omega_{s} \tau_{2}\right) \cos \left(\theta_{20}\right)-\frac{\gamma_{2}}{4 \omega_{c}} a_{10} a_{20} \sin \left(\omega_{c} \tau_{3}-\omega_{s} \tau_{2}\right) \sin \left(\theta_{20}\right)\right], \\
& r_{41}=\left[\frac{\gamma_{2}}{2 \omega_{c}} \cos \left(\omega_{c} \tau_{3}-\omega_{s} \tau_{2}\right) \cos \left(\theta_{20}\right)-\frac{\gamma_{2}}{2 \omega_{c}} \sin \left(\omega_{c} \tau_{3}-\omega_{s} \tau_{2}\right) \sin \left(\theta_{20}\right)-\frac{\sigma_{1}}{a_{10}}+\left(\frac{9 \alpha}{8 \omega_{s}}+\frac{3 \beta \omega_{s}}{8}\right) a_{10}\right], \\
& r_{42}=\left[\frac{f}{2 a_{10} \omega_{s}} \sin \left(\theta_{10}\right)\right] \text {, } \\
& r_{43}=\left[\begin{array}{l}
\frac{\left(\sigma_{2}+\sigma_{1}\right)}{a_{20}}+\frac{\gamma_{2}}{2 \omega_{c} a_{20}} a_{10} \cos \left(\omega_{c} \tau_{3}-\omega_{s} \tau_{2}\right) \cos \left(\theta_{20}\right)-\frac{\gamma_{2}}{2 \omega_{c} a_{20}} a_{10} \sin \left(\omega_{c} \tau_{3}-\omega_{s} \tau_{2}\right) \sin \left(\theta_{20}\right) \\
-\frac{\gamma_{1} a_{20}}{2 a_{10} \omega_{s}} \cos \left(2 \omega_{c} \tau_{1}\right) \cos \left(\theta_{20}\right)+\frac{\gamma_{1} a_{20}}{2 a_{10} \omega_{s}} \sin \left(2 \omega_{c} \tau_{1}\right) \sin \left(\theta_{20}\right)
\end{array}\right], \\
& r_{44}=\left[\begin{array}{l}
-\frac{\gamma_{2} a_{10}}{2 \omega_{c}} \cos \left(\omega_{c} \tau_{3}-\omega_{s} \tau_{2}\right) \sin \left(\theta_{20}\right)-\frac{\gamma_{2} a_{10}}{2 \omega_{c}} \sin \left(\omega_{c} \tau_{3}-\omega_{s} \tau_{2}\right) \cos \left(\theta_{20}\right) \\
+\frac{\gamma_{1} a_{20}^{2}}{4 a_{10} \omega_{s}} \cos \left(2 \omega_{c} \tau_{1}\right) \sin \left(\theta_{20}\right)+\frac{\gamma_{1} a_{20}^{2}}{4 a_{10} \omega_{s}} \sin \left(2 \omega_{c} \tau_{1}\right) \cos \left(\theta_{20}\right)
\end{array}\right] \\
& \zeta_{1}=-r_{11}-r_{22}-r_{33}-r_{44} \\
& \zeta_{2}=r_{33} r_{44}-r_{21} r_{12}+r_{11} r_{44}-r_{42} r_{24}+r_{11} r_{33}-r_{31} r_{13}-r_{41} r_{14}-r_{32} r_{23}-r_{34} r_{43}+r_{11} r_{22}+r_{22} r_{33}+r_{22} r_{44} \\
& \zeta_{3}=r_{31} r_{12} r_{23}+r_{31} r_{13} r_{44}+r_{32} r_{23} r_{44}+r_{11} r_{32} r_{23}+r_{22} r_{34} r_{43}+r_{32} r_{43} r_{24}+r_{31} r_{43} r_{14}+r_{21} r_{12} r_{44} \\
& +r_{21} r_{12} r_{33}+r_{11} r_{42} r_{24}-r_{11} r_{22} r_{44}-r_{11} r_{22} r_{33}+r_{21} r_{32} r_{13}+r_{31} r_{22} r_{13}+r_{41} r_{12} r_{24}+r_{21} r_{42} r_{14} \\
& -r_{22} r_{33} r_{44}+r_{41} r_{14} r_{33}+r_{41} r_{13} r_{34}+r_{41} r_{22} r_{14}+r_{42} r_{23} r_{34}+r_{42} r_{24} r_{33}-r_{11} r_{33} r_{44}+r_{11} r_{34} r_{43} \\
& \zeta_{4}=r_{11} r_{22} r_{33} r_{44}-r_{11} r_{22} r_{34} r_{43}-r_{11} r_{32} r_{43} r_{24}-r_{11} r_{32} r_{23} r_{44}-r_{11} r_{42} r_{23} r_{34}-r_{11} r_{42} r_{24} r_{33} \\
& -r_{21} r_{12} r_{33} r_{44}+r_{21} r_{12} r_{34} r_{43}-r_{21} r_{32} r_{43} r_{14}-r_{21} r_{32} r_{13} r_{44}-r_{21} r_{42} r_{13} r_{34}-r_{21} r_{42} r_{14} r_{33} \\
& -r_{31} r_{12} r_{23} r_{44}-r_{31} r_{12} r_{43} r_{24}-r_{31} r_{22} r_{43} r_{14}-r_{31} r_{22} r_{13} r_{44}+r_{31} r_{42} r_{13} r_{24}-r_{31} r_{42} r_{23} r_{14} \\
& -r_{41} r_{12} r_{23} r_{34}-r_{41} r_{12} r_{24} r_{33}-r_{41} r_{22} r_{14} r_{33}-r_{41} r_{22} r_{13} r_{34}-r_{41} r_{32} r_{13} r_{24}+r_{41} r_{32} r_{23} r_{14}
\end{aligned}
$$

